\title{
Nonlinear Disturbance-Observer-Based Sliding Mode Control for Flexible Air-Breathing Hypersonic Vehicles
}

\author{
Na Wang, ${ }^{1}$ Xiu-Ming Yao, ${ }^{2}$ and Wen-Shuo $\mathrm{Li}^{3}$ \\ ${ }^{1}$ School of Instrumentation Science and Opto-Electronics Engineering, Beihang University, Xue Yuan Road No. 37, \\ Haidian District, Beijing 100191, China \\ ${ }^{2}$ National Key Laboratory on Aircraft Control Technology, Beihang University, Xue Yuan Road No. 37, Haidian District, \\ Beijing 100191, China \\ ${ }^{3}$ School of Automation Science and Electrical Engineering, Beihang University, Xue Yuan Road No. 37, Haidian District, \\ Beijing 100191, China
}

Correspondence should be addressed to Na Wang; wangnaflcon@126.com

Received 10 July 2014; Accepted 2 October 2014

Academic Editor: Xudong Zhao

Copyright (c) $2015 \mathrm{Na}$ Wang et al. This is an open access article distributed under the Creative Commons Attribution License, which permits unrestricted use, distribution, and reproduction in any medium, provided the original work is properly cited.

\begin{abstract}
This paper investigates a tracking problem for flexible air-breathing hypersonic vehicles (FAHVs) with composite disturbance. The composite disturbance produced by flexible effects, parameter uncertainties, and external interferences is modeled as a kind of unknown derivative-bounded disturbance in this paper. Then a novel composite control strategy is presented for the nonlinear FAHV model with the composite disturbance, which combines a nonlinear disturbance-observer-based compensator (NDOBC) and a dynamic-inversion-based sliding mode controller (DIBSMC). Specifically, the NDOBC is constructed to estimate and compensate for the composite disturbance, and the DIBSMC is designed to track desired trajectories of velocity and flight path angle. Moreover, the uniformly ultimate boundedness of the composite system can be guaranteed by using Lyapunov theory. Finally, simulation results on a full nonlinear model of FAHVs demonstrate that the proposed nonlinear disturbance-observer-based sliding mode controller is more effective than the traditional DIBSMC. Specifically, it is shown that the chattering of traditional DIBSMC in presence of composite disturbances can be attenuated with the NDOBC.
\end{abstract}

\section{Introduction}

Flight control of air-breathing hypersonic vehicles (AHVs) is very important and difficult. AHVs use scramjet engines integrated with the airframe, so the vehicle dynamics display strong interactions between the elastic airframe, the propulsion system, and the structural dynamics (see [1-3] and references therein). In addition, significant flexible effects cannot be neglected in the control design due to the slender geometries and light structures of AHVs (see [4-6] and references therein). It has been shown that these strong couplings, undesired flexible effects, large parametric uncertainties, and various external disturbances may cause degradation of the performance of flight control systems. Thus, the desired control scheme should be robust enough to overcome these factors.

In the past few years, many effective robust control laws have been presented (see, e.g., [7-13]) for a nonlinear AHV model developed at NASA Langley Research Center. For example, an observer-based passive fault-tolerant control scheme was successfully applied for this AHV model with both parameter uncertainty and actuator faults in [13]. But only rigid modes were considered in the nonlinear AHV model. Thus a new FAHV model was developed in [6], which includes not only the interactions between the propulsion system and the airframe dynamics but also the strong flexible effects. After that, many nonlinear controllers have been applied effectively to this nonlinear model developed by 
Parker et al. and Fiorentini et al. (see, e.g., [14-18]). An effective adaptive sliding mode controller was designed for the linearized FAHV model in [15]. But the capability of the linear model to represent the dynamics and the coupling effects is limited. So nonlinear control methods were concerned for the nonlinear FAHV model in $[16,17]$. However, it was based on control-design models without any consideration of rigidflexible coupling dynamics. Due to these couplings, rigidbody state and control input will be affected by flexible modes. In fact, it has been shown that the undesired flexible effects may cause degradation to the performance of flight control systems (see $[6,18]$ ). Therefore, the flexible effects were studied and transformed into elastic-mode-related terms that appeared in forces and moment in [18]. However, it may lead to a large amount of online calculations because many coefficients have to be estimated using adaptive methods. To avoid these problems, a novel nonlinear disturbanceobserver-based sliding mode control strategy was proposed to achieve control objective and attenuate the composite disturbance produced by flexible effects, parameter uncertainties, and external interferences in this paper.

Recently, the disturbance-observer-based control (DOBC) method has attracted considerable attention as a robust control scheme. Many effective DOBC schemes have been developed for spacecraft, missiles, and hypersonic vehicles (see, e.g., [19-23]). Chen firstly designed a dynamic inversion controller based on disturbance observer for missile systems in [20]. An antidisturbance PD control scheme was successfully applied for the attitude control of flexible spacecrafts in [21]. Chen et al. proposed a robust control law using disturbance observer and neural networks for the linearized AHV model in [22]. Li et al. presented a composite controller based on disturbance observer for the nonlinear AHV model in [23]. However, the study for the nonlinear FAHV model with composite disturbance using DOBC strategy is still insufficient. In this paper, a novel composite control strategy, which combines a nonlinear disturbance-observer-based compensator (NDOBC) and a dynamic-inversion-based sliding mode controller (DIBSMC), is presented for the nonlinear FAHV model with composite disturbance.

In conclusion, the main contributions in this paper can be summarized as follows. (1) The composite disturbance produced by flexible effects, parameter uncertainties, and external interferences is formulated as a kind of unknown disturbance, which is considered in the control-oriented model and estimated by a nonlinear disturbance observer. (2) Combining a NDOBC with a DIBSMC, a nonlinear disturbanceobserver-based sliding mode controller (NDOBSMC) is proposed to make velocity and flight-path angle track desired signals and reject the composite disturbance. (3) The stability of composite closed-loop system which includes two trackingerror equations and a disturbance estimation error equation is analyzed.

The remainder of this paper is organized as follows. In Section 2, the nonlinear FAHV model with composite disturbance is introduced. In Section 3, the NDOBC and the NDOBSMC are designed, and stability of the composite system is analyzed. In Section 4, the effectiveness of the proposed NDOBSMC is confirmed through numerical simulations. Conclusions are provided in Section 5.

\section{Problem Formulation}

A nonlinear model for the longitudinal dynamics of flexible air-breathing hypersonic vehicles, as given by Bolender and Doman in [6], is described by the following nonlinear equations:

$$
\begin{gathered}
\dot{h}=V \sin (\theta-\alpha), \\
\dot{V}=\frac{T \cos \alpha-D}{m}-g \sin (\theta-\alpha), \\
\dot{\alpha}=-\frac{L+T \sin \alpha}{m V}+Q+\frac{g}{V} \cos (\theta-\alpha), \\
I_{y y} \dot{Q}=M+\widetilde{\psi}_{1} \ddot{\eta}_{1}+\widetilde{\psi}_{2} \ddot{\eta}_{2}+\bar{d}_{o}, \\
K_{1} \ddot{\eta}_{1}=-2 \zeta_{1} \omega_{1} \dot{\eta}_{1}-\omega_{1}^{2} \eta_{1}+N_{1}-\frac{\left(\widetilde{\psi}_{1} M\right)}{I_{y y}}-\frac{\left(\widetilde{\psi}_{1} \widetilde{\psi}_{2} \ddot{\eta}_{2}\right)}{I_{y y}}, \\
K_{2} \ddot{\eta}_{2}=-2 \zeta_{2} \omega_{2} \dot{\eta}_{2}-\omega_{2}^{2} \eta_{2}+N_{2}-\frac{\left(\widetilde{\psi}_{2} M\right)}{I_{y y}}-\frac{\left(\widetilde{\psi}_{1} \widetilde{\psi}_{2} \ddot{\eta}_{1}\right)}{I_{y y}},
\end{gathered}
$$

where $\widetilde{\psi}_{1}=\int_{-L_{f}}^{0} \widehat{m}_{f} \phi_{f}(\xi) d \xi, \widetilde{\psi}_{2}=\int_{0}^{L_{a}} \widehat{m}_{a} \phi_{a}(\xi) d \xi, K_{1}=1+$ $\widetilde{\psi}_{1}^{2} / I_{y y}$, and $K_{2}=1+\widetilde{\psi}_{2}^{2} / I_{y y}$, in which $\phi_{f}(\xi)$ and $\phi_{a}(\xi)$ are the mode shapes and $\widehat{m}_{f}$ and $\widehat{m}_{a}$ are mass densities. It is noted that a pitching disturbance moment, $\bar{d}_{o}$, is considered in this paper, which may originate from parameter uncertain, sensor noise, actuator error, and external disturbance.

The approximations of the forces and moment given by Parker et al. in [17] are described as follows:

$$
\begin{aligned}
& L=\bar{q} S\left(C_{L}^{\alpha} \alpha+C_{L}^{0}+C_{L}^{\delta_{e}} \delta_{e}\right), \\
& D=\bar{q} S\left(C_{D}^{\alpha^{2}} \alpha^{2}+C_{D}^{\alpha} \alpha+C_{D}^{0}+C_{D}^{\delta_{e}^{2}} \delta_{e}^{2}+C_{D}^{\delta_{e}} \delta_{e}\right), \\
& T=\left(C_{T \Phi}^{\alpha^{3}} \alpha^{3}+C_{T \Phi}^{\alpha^{2}} \alpha^{2}+C_{T \Phi}^{\alpha} \alpha+C_{T \Phi}^{0}\right) \Phi \\
& \quad+C_{T}^{\alpha^{3}} \alpha^{3}+C_{T}^{\alpha^{2}} \alpha^{2}+C_{T}^{\alpha} \alpha+C_{T}^{0}, \\
& M=z_{T}^{T}+\bar{q} S \bar{c}\left(C_{M}^{\alpha^{2}} \alpha^{2}+C_{M}^{\alpha} \alpha+C_{M}^{0}+C_{M}^{\delta_{e}} \delta_{e}\right), \\
& N_{1}=C_{N_{1}}^{\alpha^{2}} \alpha^{2}+C_{N_{1}}^{\alpha} \alpha+C_{N_{1}}^{0}, \\
& N_{2}=C_{N_{2}}^{\alpha^{2}} \alpha^{2}+C_{N_{2}}^{\alpha} \alpha+C_{N_{2}}^{0}+C_{N_{2}}^{\delta_{e}} \delta_{e} .
\end{aligned}
$$

Coefficients that appear in the forces and moment (i.e., $C_{L}^{(\cdot)}, C_{D}^{(\cdot)}, C_{M}^{(\cdot)}, C_{T}^{(\cdot)}, C_{T \Phi}^{(\cdot)}$, and $C_{N_{i}}^{(\cdot)}$ ) are subject to uncertainty in this paper. Denote $C_{L^{0}}^{(\cdot)}, C_{D^{0}}^{(\cdot)}, C_{M^{0}}^{(\cdot)}, C_{T^{0}}^{(\cdot)}, C_{T^{0} \Phi}^{(\cdot)}$, and $C_{N_{i}^{0}}^{(\cdot)}$ as nominal values of the coefficients. The uncertainty is modeled as an additive variable $\Delta$ to the nominal values used for control design; then the coefficients can be expressed as 
$C_{L}^{(\cdot)}=C_{L^{0}}^{(\cdot)}\left(1+\Delta C_{L}^{(\cdot)}\right), C_{D}^{(\cdot)}=C_{D^{0}}^{(\cdot)}\left(1+\Delta C_{D}^{(\cdot)}\right), C_{M}^{(\cdot)}=C_{M^{0}}^{(\cdot)}(1+$ $\left.\Delta C_{M}^{(\cdot)}\right), C_{T}^{(\cdot)}=C_{T^{0}}^{(\cdot)}\left(1+\Delta C_{T}^{(\cdot)}\right), C_{T \Phi}^{(\cdot)}=C_{T^{0} \Phi}^{(\cdot)}\left(1+\Delta C_{T \Phi}^{(\cdot)}\right)$, and $C_{N_{i}}^{(\cdot)}=C_{N_{i}^{0}}^{(\cdot)}\left(1+\Delta C_{N_{i}}^{(\cdot)}\right)$. The forces and moment are given by $L=L^{0}+\Delta L, D=D^{0}+\Delta D, T=T^{0}+\Delta T, M=M^{0}+\Delta M$, and $N_{i}=N_{i}^{0}+\Delta N_{i}$, where the detailed expressions of $L^{0}, D^{0}$, $T^{0}, N_{i}^{0}, M^{0}, \Delta L, \Delta L, \Delta D, \Delta T, \Delta N_{i}$, and $\Delta M$ are given in the appendix.

In this paper, the control design problem is to select control inputs, $\delta_{e}$ and $\Phi$, that force the outputs, $V$ and $\gamma$, to track commanded values, $V_{d}$ and $\gamma_{d}$, in the presence of the composite disturbance produced by the couplings, uncertainties, and external interferences.

\section{Composite Controller Design}

This section presents a novel composite controller with a hierarchical architecture for the nonlinear FAHV model. Firstly, a nonlinear control-oriented model (COM) with composite disturbance is provided. Secondly, a nonlinear disturbance observer (NDO) based compensator (NDOBC) is constructed to estimate and compensate for the composite disturbance. Then a NDO-based sliding mode controller (NDOBSMC) is designed to track the desired trajectories in the presence of composite disturbance. Finally, stability of the composite system is analyzed.

3.1. COM with Composite Disturbance. In this part, a novel $\mathrm{COM}$ with composite disturbance is obtained from the FAHV nonlinear model (1) by removing the altitude dynamic and flexible modes and setting the weak elevator couplings to zero. The new COM is given by the following set of equations:

$$
\begin{gathered}
\dot{V}=\frac{T^{0} \cos \alpha-\bar{D}^{0}}{m}-g \sin \gamma, \\
\dot{\alpha}=-\frac{\bar{L}^{0}+T^{0} \sin \alpha}{m V}+Q+\frac{g}{V} \cos \gamma, \\
\dot{\gamma}=\frac{\bar{L}^{0}+T^{0} \sin \alpha}{m V}-\frac{g}{V} \cos \gamma, \\
\bar{I}_{y y} \dot{Q}=M^{0}+\widetilde{\psi}_{1} N_{1}^{0}+\widetilde{\psi}_{2} N_{2}^{0}+\bar{d}, \\
\ddot{\Phi}=-2 \zeta \omega \dot{\Phi}-\omega^{2} \Phi+\omega^{2} \Phi_{c},
\end{gathered}
$$

where $\bar{I}_{y y}=I_{y y}-\widetilde{\psi}_{1}^{2}-\widetilde{\psi}_{2}^{2}, \bar{L}^{0}=\bar{q} S\left(C_{L^{0}}^{\alpha} \alpha+C_{L^{0}}^{0}\right)$, and $\bar{D}^{0}=$ $\bar{q} S\left(C_{D^{0}}^{\alpha^{2}} \alpha^{2}+C_{D^{0}}^{\alpha} \alpha+C_{D^{0}}^{0}\right)$. Besides, the second-order dynamic equation for fuel equivalence ratio, $\Phi$, is added to obtain the COM with full vector relative degree. In addition, the composite disturbance, $\bar{d}$, is considered in this COM, which contains $\bar{d}_{o}$ and a pitching disturbance moment, $\bar{d}_{f}$, produced by the couplings between flexible and rigid modes. Denote $d=\bar{d} / \bar{I}_{y y}$ in order to simplify the following design of disturbance observer.
Remark 1. It is different from [17] that the composite disturbance, $\bar{d}$, produced by parameter uncertainties, external disturbances, and couplings between rigid and flexible modes is considered in the proposed COM.

3.2. NDOBC Design. The nonlinear COM described by (3) is a special case of the generic muti-input/multioutput (MIMO) nonlinear system:

$$
\begin{gathered}
\dot{x}=f(x)+g_{1}(x) u+g_{2}(x) d, \\
y=h(x),
\end{gathered}
$$

where state $x=\left[\begin{array}{llllll}V & \alpha & \gamma & Q & \Phi & \Phi\end{array}\right]^{T}$, control input $u=$ $\left[\begin{array}{ll}\delta_{e} & \Phi_{c}\end{array}\right]^{T}$, output $y=\left[\begin{array}{ll}V & \gamma\end{array}\right]^{T}$, and composite disturbance $d=\bar{d} / \bar{I}_{y y}$. Nonlinear functions $f, g_{1}$, and $g_{2}$ are sufficiently smooth with respect to $x \in \mathscr{R}^{6}$, and the concrete forms of $f$, $g_{1}$, and $g_{2}$ are given by

$$
\begin{aligned}
& f(x)=\left[\begin{array}{llllll}
f_{1}(x) & f_{2}(x) & f_{3}(x) & f_{4}(x) & f_{5}(x) & f_{6}(x)
\end{array}\right]^{T}, \\
& g_{1}(x)=\left[\begin{array}{lll}
g_{11} & (x) & g_{12}(x)
\end{array}\right] \\
& g_{2}(x)=\left[\begin{array}{llllll}
0 & 0 & 0 & 1 & 0 & 0
\end{array}\right]^{T},
\end{aligned}
$$

in which

$$
\begin{aligned}
& f_{1}(x)=\frac{T^{0} \cos \alpha-\bar{D}^{0}}{m}-g \sin \gamma, \\
& f_{2}(x)=-\frac{\bar{L}^{0}+T^{0} \sin \alpha}{m V}+Q+\frac{g}{V} \cos \gamma, \\
& f_{3}(x)=\frac{\bar{L}^{0}+T^{0} \sin \alpha}{m V}-\frac{g}{V} \cos \gamma, \\
& f_{4}(x) \\
& =\frac{\left[z_{T} T^{0}+\bar{q} S \bar{c}\left(C_{M^{0}}^{\alpha^{2}} \alpha^{2}+C_{M^{0}}^{\alpha} \alpha+C_{M^{0}}^{0}\right)+\widetilde{\psi}_{1} N_{1}^{0}+\widetilde{\psi}_{2} \bar{N}_{2}^{0}\right]}{\bar{I}_{y y}}, \\
& f_{5}(x)=\dot{\Phi}, \\
& f_{6}(x)=-2 \zeta \omega \dot{\Phi}-\omega^{2} \Phi,
\end{aligned}
$$

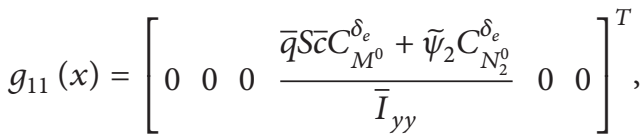

$$
\begin{aligned}
& g_{12}(x)=\left[\begin{array}{llllll}
0 & 0 & 0 & 0 & 0 & \omega^{2}
\end{array}\right]^{T}, \\
& \bar{N}_{2}^{0}=N_{2}^{0}-C_{N_{2}^{0}}^{\delta_{e}} \delta_{e} .
\end{aligned}
$$


For the MIMO nonlinear system (4), a NDO is designed to estimate the unknown composite disturbance $d$ according to [20], which is given by

$$
\begin{gathered}
\hat{d}=z+p(x), \\
\dot{z}=-l(x) g_{2}(x) z-l(x)\left(g_{2}(x) p(x)+f(x)+g_{1}(x) u\right),
\end{gathered}
$$

where $\widehat{d}$ and $z$ are the estimate of the unknown disturbance and the internal state of the nonlinear observer, respectively. Besides, $p(x)$ is a nonlinear function to be designed and the nonlinear observer gain $l(x)$ is defined by

$$
\begin{aligned}
l(x) & =\frac{\partial p(x)}{\partial x} \\
& =\left[\begin{array}{lllllll}
l_{1}(x) & l_{2}(x) & l_{3}(x) & l_{4}(x) & l_{5}(x) & l_{6}(x)
\end{array}\right] .
\end{aligned}
$$

Define the estimation error by $e_{d}(t)=d-\widehat{d}$. The error dynamic is described as

$$
\dot{e}_{d}(t)+l(x) g_{2}(x) e_{d}(t)=\dot{d},
$$

where $\dot{d}$ is the first derivative of composite disturbance $d$. It is assumed that $|\dot{d}| \leq \sigma$ in this paper, where $\sigma$ is a known positive constant.

Substituting $g_{2}(x)$ and $l(x)$ into (9), the differential equation (9) can be rewritten as

$$
\dot{e}_{d}(t)+l_{4}(x) e_{d}(t)=\dot{d},
$$

where $l_{4}(x)=\partial p(x) / \partial Q$. An appropriate fourth element of observer gain, $l_{4}(x)$, needs to be designed such that $e_{d}(t)$ converge to zero.

The following result provides a design scheme for the disturbance observer (7) with guaranteed uniformly ultimate boundedness of system (9) or (10).

Lemma 2 (for details see [24]). Let $\mathscr{D} \subset \mathscr{R}^{n}$ be a domain that contains the origin and let $W:[0, \infty) \times \mathscr{D} \rightarrow \mathscr{R}$ be a continuously differentiable function such that

$$
\begin{gathered}
\alpha_{1}(\|\chi\|) \leq W(t, \chi) \leq \alpha_{2}(\|\chi\|), \\
\frac{\partial W}{\partial t}+\frac{\partial W}{\partial \chi} \xi(t, \chi) \leq-Q(\chi), \quad\|\chi\| \geq \mu>0,
\end{gathered}
$$

for all $t \geq 0$ and for all $\chi \in \mathscr{D}$, where $\alpha_{1}$ and $\alpha_{2}$ are class $\mathscr{K}$ functions and $Q(\chi)$ is a continuous positive definite function. Then, there is $T \geq 0$ (dependent on $\chi\left(t_{0}\right)$ and $\mu$ ) such that the solution of the system

$$
\dot{\chi}=\xi(t, \chi)
$$

satisfies

$$
\|\chi(t)\| \leq \alpha_{1}^{-1}\left(\alpha_{2}(\mu)\right), \quad \forall t \geq t_{0}+T .
$$

Moreover, if $\mathscr{D}=\mathscr{R}^{n}$ and $\alpha_{1}$ belongs class $\mathscr{K}_{\infty}$, then (13) holds for any initial state $\chi\left(t_{0}\right)$, with one restriction on how large $\mu$ is. Note that inequality (13) shows that $\chi(t)$ is uniformly ultimately bounded (UUB) with the ultimate bound $\alpha_{1}^{-1}\left(\alpha_{2}(\mu)\right)$.
Theorem 3. For the estimation error system (10), if there exists a constant $l_{0}$ satisfying

$$
l_{0}>\frac{1}{[2(1+\underline{M})]},
$$

where the fourth element of observer gain, $l_{4}(x)$, is chosen as $l_{0}(1+M(x))$ and $M(x)$ is a bounded nonlinear function with respect to $x$ satisfying $0 \leq \underline{M} \leq M(x) \leq \bar{M}$, then the solution of system (10) is UUB.

Proof. Denote the Lyapunov function

$$
W_{d}(t)=e_{d}^{2}(t)
$$

Computing the derivative of $W_{d}(t)$ along the trajectories of (10), it can be obtained that

$$
\begin{aligned}
\dot{W}_{d}(t)= & 2 e_{d}(t) \dot{e}_{d}(t)=2 e_{d}(t)\left[-l_{0}(1+M(x)) e_{d}(t)+\dot{d}\right] \\
= & -2 l_{0}(1+M(x)) e_{d}^{2}(t)-\left(e_{d}^{2}(t)-2 e_{d}(t) \dot{d}+\dot{d}^{2}\right) \\
& +\left(e_{d}^{2}(t)+\dot{d}^{2}\right) \\
\leq & -\left[2 l_{0}(1+M(x))-1\right] e_{d}^{2}(t)+\sigma^{2} .
\end{aligned}
$$

Thus it can be concluded from Lemma 2 that the solution of estimation error system (10) is uniformly ultimately bounded, if $l_{0}>1 /[2(1+\underline{M})]$. Obviously, there exits a positive constant $\beta$ such that $(1 /(1+\beta)) e_{d}^{2}(t) \leq W_{d}(t) \leq(1+\beta) e_{d}^{2}(t)$. And $\dot{W}_{d}(t)$ is a negative definite matrix when $\left|e_{d}(t)\right| \geq \sigma>0$ hold. According to Lemma 2, the ultimate bound of $e_{d}(t)$ is $(1+\beta) \sigma$; that is, $\left|e_{d}(t)\right| \leq(1+\beta) \sigma$.

Remark 4. The NDO (7) reduces to a linear disturbance observer (LDO) if $M(x)=0$. Obviously, the performance of NDO is better than LDO when LDO takes the same constant $l_{0}$ as NDO.

3.3. NDOBSMC Design. The nonlinear COM described by (3) can be linearized completely using the input/output linearization technique of full state feedback, so the linearized model is developed by repeated differentiation of $V$ and $\gamma$ as follows:

$$
\begin{aligned}
& \dddot{V}=\frac{\sigma_{1} \ddot{x}_{1}+\dot{x}_{1}^{T} \Sigma_{2} \dot{x}_{1}}{m}, \\
& \dddot{\gamma}=\pi_{1} \ddot{x}_{1}+\dot{x}_{1}^{T} \Pi_{2} \dot{x}_{1},
\end{aligned}
$$

where $x_{1}=\left[\begin{array}{llll}V & \alpha & \gamma & \Phi\end{array}\right]^{T}, \sigma_{1}=\partial f_{1}(x) / \partial x_{1}, \Sigma_{2}=\partial \sigma_{1} / \partial x_{1}$, $\pi_{1}=\partial f_{3}(x) / \partial x_{1}$, and $\Pi_{2}=\partial \pi_{1} / \partial x_{1}$. The detailed expressions of $\sigma_{1}, \Sigma_{2}, \pi_{1}$, and $\Pi_{2}$ are given in the appendix.

The right-hand sides of (17) involve second derivatives of $\alpha$ and $\Phi$. The expression of the second derivatives for $\alpha$ and $\Phi$ consists of three parts: the first part is control relevant, 
the second part is disturbance relevant, and the third part is neither control relevant nor disturbance relevant. Consider

$$
\begin{gathered}
\ddot{\alpha}=\ddot{\alpha}_{0}+\frac{\bar{q} S \bar{c} C_{M^{0}}^{\delta_{e}}+\widetilde{\psi}_{2} C_{N_{2}^{0}}^{\delta_{e}}}{\bar{I}_{y y}}+d, \\
\ddot{\Phi}=\ddot{\Phi}_{0}+\omega^{2} \Phi_{c},
\end{gathered}
$$

where $\ddot{\alpha}_{0}=\left[z_{T} T^{0}+\bar{q} S \bar{c}\left(C_{M^{0}}^{\alpha^{2}} \alpha^{2}+C_{M^{0}}^{\alpha} \alpha+C_{M^{0}}^{0}\right)+\widetilde{\psi}_{1} N_{1}^{0}+\right.$ $\left.\widetilde{\psi}_{2} \bar{N}_{2}^{0}\right] / \bar{I}_{y y}-\ddot{\gamma}$ and $\ddot{\Phi}_{0}=-2 \zeta \omega \dot{\Phi}-\omega^{2} \Phi$.

When $x_{10}=\left[\begin{array}{llll}V & \alpha_{0} & \gamma & \Phi_{0}\end{array}\right]^{T}$ is defined, the output dynamics of $V$ and $\gamma$ can be written as follows:

$$
\left[\begin{array}{c}
\dddot{V} \\
\dddot{\gamma}
\end{array}\right]=\left[\begin{array}{l}
f_{V} \\
f_{\gamma}
\end{array}\right]+B\left(x_{1}\right)\left[\begin{array}{l}
\delta_{e} \\
\Phi_{c}
\end{array}\right]+\left[\begin{array}{l}
c_{V}\left(x_{1}\right) \\
c_{\gamma}\left(x_{1}\right)
\end{array}\right] d+\left[\begin{array}{l}
d_{V} \\
d_{\gamma}
\end{array}\right],
$$

where control inputs, $\delta_{e}$ and $\Phi_{c}$, and composite disturbance, $d$, appear explicitly. Disturbances, $d_{V}$ and $d_{\gamma}$, are produced by parameter uncertainties. The concrete forms of $f_{V}, f_{\gamma}, B\left(x_{1}\right)$, $c_{V}\left(x_{1}\right)$, and $c_{\gamma}\left(x_{1}\right)$ are given by

$$
\begin{gathered}
f_{V}=\frac{\sigma_{1} \ddot{x}_{1}+\dot{x}_{1}^{T} \Sigma_{2} \dot{x}_{1}}{m}, \\
f_{\gamma}=\pi_{1} \ddot{x}_{1}+\dot{x}_{1}^{T} \Pi_{2} \dot{x}_{1}, \\
B\left(x_{1}\right)=\left[\begin{array}{ll}
b_{11}\left(x_{1}\right) & b_{12}\left(x_{1}\right) \\
b_{21}\left(x_{1}\right) & b_{22}\left(x_{1}\right)
\end{array}\right], \\
c_{V}\left(x_{1}\right)=\frac{1}{m}\left(\frac{\partial T^{0}}{\partial \alpha} \cos \alpha-T^{0} \sin \alpha-\frac{\partial \bar{D}^{0}}{\partial \alpha}\right), \\
c_{\gamma}\left(x_{1}\right)=\frac{1}{m V}\left(\frac{\partial T^{0}}{\partial \alpha} \sin \alpha+T^{0} \cos \alpha-\frac{\partial \bar{L}}{\partial \alpha}\right),
\end{gathered}
$$

in which

$$
\begin{aligned}
b_{11}\left(x_{1}\right)= & \left(\frac{\bar{q} S \bar{c} C_{M^{0}}^{\delta_{e}}+\widetilde{\psi}_{2} C_{N_{2}^{0}}^{\delta_{e}}}{m I_{y y}}\right) \\
& \times\left(\frac{\partial T^{0}}{\partial \alpha} \cos \alpha-T^{0} \sin \alpha-\frac{\partial \bar{D}^{0}}{\partial \alpha}\right), \\
b_{12}\left(x_{1}\right)= & \left(\frac{\omega^{2}}{m}\right)\left(\frac{\partial T^{0}}{\partial \Phi} \cos \alpha\right), \\
b_{21}\left(x_{1}\right)= & \left(\frac{\bar{q} S \bar{c} C_{M^{0}}^{\delta_{e}}+\widetilde{\psi}_{2} C_{N_{2}^{0}}^{\delta_{e}}}{m V I_{y y}}\right) \\
& \times\left(\frac{\partial T^{0}}{\partial \alpha} \sin \alpha+T^{0} \cos \alpha-\frac{\partial \bar{L}^{0}}{\partial \alpha}\right), \\
b_{22}\left(x_{1}\right)= & \left(\frac{\omega^{2}}{m V}\right)\left(\frac{\partial T^{0}}{\partial \Phi} \sin \alpha\right) .
\end{aligned}
$$

Two decoupled sliding mode surfaces $s_{V}(t)$ and $s_{\gamma}(t)$ are defined by $s_{V}(t)=\left((d / d t)+\lambda_{V}\right)^{3} \int_{0}^{t} e_{V}(\tau) d \tau$ and $s_{\gamma}(t)=$ $\left((d / d t)+\lambda_{\gamma}\right)^{3} \int_{0}^{t} e_{\gamma}(\tau) d \tau$, where $e_{V}(t)=V-V_{d}$ is tracking error of velocity, $e_{\gamma}(t)=\gamma-\gamma_{d}$ is tracking error of flight path angle, and $\lambda_{V}$ and $\lambda_{\gamma}$ are strictly positive constants defining the bandwidth of the tracking error dynamics. The sliding mode surfaces $s_{V}(t)$ and $s_{\gamma}(t)$ represent linear differential equations where their solutions $\int e_{V}(\tau)$ and $\int e_{\gamma}(\tau)$ converge to zero exponentially with the time constants $2 / \lambda_{V}$ and $2 / \lambda_{\gamma}$, respectively.

Differentiating $s_{V}(t)$ and $s_{\gamma}(t)$, we have

$$
\begin{aligned}
{\left[\begin{array}{c}
\dot{s}_{V}(t) \\
\dot{s}_{\gamma}(t)
\end{array}\right]=} & {\left[\begin{array}{l}
v_{V} \\
v_{\gamma}
\end{array}\right]+\left[\begin{array}{l}
f_{V} \\
f_{\gamma}
\end{array}\right]+B\left(x_{1}\right)\left[\begin{array}{l}
\delta_{e} \\
\Phi_{c}
\end{array}\right] } \\
& +\left[\begin{array}{l}
c_{V}\left(x_{1}\right) \\
c_{\gamma}\left(x_{1}\right)
\end{array}\right] d+\left[\begin{array}{l}
d_{V} \\
d_{\gamma}
\end{array}\right],
\end{aligned}
$$

where $v_{V}=-\dddot{V}_{d}+3 \lambda_{V} \ddot{e}_{V}(t)+3 \lambda_{V}^{2} \dot{e}_{V}(t)+\lambda_{V}^{3} e_{V}(t)$ and $v_{\gamma}=$ $-\dddot{\gamma}_{d}+3 \lambda_{\gamma} \ddot{e}_{\gamma}(t)+3 \lambda_{\gamma}^{2} \dot{e}_{\gamma}(t)+\lambda_{\gamma}^{3} e_{\gamma}(t)$

With (22), recall that estimation value of the disturbance $d$ is developed by the NDO (7) and the NDOBSMC can be designed as

$$
\begin{aligned}
{\left[\begin{array}{l}
\delta_{e} \\
\Phi_{c}
\end{array}\right]=} & -B^{-1}\left(x_{1}\right)\left[\begin{array}{l}
f_{V} \\
f_{\gamma}
\end{array}\right]-B^{-1}\left(x_{1}\right)\left[\begin{array}{l}
v_{V}+k_{V} \operatorname{sgn}\left(s_{V}(t)\right) \\
v_{\gamma}+k_{\gamma} \operatorname{sgn}\left(s_{\gamma}(t)\right)
\end{array}\right] \\
& -B^{-1}\left(x_{1}\right)\left[\begin{array}{l}
c_{V}\left(x_{1}\right) \\
c_{\gamma}\left(x_{1}\right)
\end{array}\right] \widehat{d},
\end{aligned}
$$

where $k_{V}$ and $k_{\gamma}$ are sliding mode gains of the NDOBSMC (23), $\widehat{d}$ is the disturbance estimation value obtained by the NDO (7), and $B\left(x_{1}\right)$ is assumed to be invertible.

Remark 5. When the output of disturbance observer $\widehat{d}=0$, that is, there is no disturbance observer, the NDOBSMC (23) obviously reduces to a DIBSMC, which is written as

$$
\left[\begin{array}{l}
\delta_{e} \\
\Phi_{c}
\end{array}\right]=-B^{-1}\left(x_{1}\right)\left[\begin{array}{l}
f_{V} \\
f_{\gamma}
\end{array}\right]-B^{-1}\left(x_{1}\right)\left[\begin{array}{l}
v_{V}+k_{V} \operatorname{sgn}\left(s_{V}(t)\right) \\
v_{\gamma}+k_{\gamma} \operatorname{sgn}\left(s_{\gamma}(t)\right)
\end{array}\right] .
$$

As discussed in [25], the control laws (23) and (24) may result in control chattering because of the discontinuity across the sliding surfaces. As a practical matter, chattering is undesirable because it involves very high control action and may excite high frequency dynamics neglected in the modeling. The discontinuity in the control law can be dealt with by defining two thin boundary layers of widths $\Phi_{V}$ and $\Phi_{\gamma}$ around the sliding mode surfaces, that is, replacing $\operatorname{sgn}\left(s_{V}(t)\right)$ and $\operatorname{sgn}\left(s_{\gamma}(t)\right)$ with continuous saturation functions $\operatorname{sat}\left(s_{V}(t) / \Phi_{V}\right)$ and $\operatorname{sat}\left(s_{\gamma}(t) / \Phi_{\gamma}\right)$, where

$$
\operatorname{sat}\left(x_{s}\right)= \begin{cases}1, & \text { if } x_{s}>1, \\ x_{s}, & \text { if }\left|x_{s}\right| \leq 1 \\ -1, & \text { if } x_{s}<-1\end{cases}
$$


So the NDOBSMC (23) and the DIBSMC (24) are modified as follows:

$$
\begin{aligned}
{\left[\begin{array}{l}
\delta_{e} \\
\Phi_{c}
\end{array}\right]=} & -B^{-1}\left(x_{1}\right)\left[\begin{array}{l}
f_{V} \\
f_{\gamma}
\end{array}\right] \\
& -B^{-1}\left(x_{1}\right)\left[\begin{array}{l}
v_{V}+k_{V} \operatorname{sat}\left(\frac{s_{V}(t)}{\Phi_{V}}\right) \\
v_{\gamma}+k_{\gamma} \operatorname{sat}\left(\frac{s_{\gamma}(t)}{\Phi_{\gamma}}\right)
\end{array}\right] \\
& -B^{-1}\left(x_{1}\right)\left[\begin{array}{l}
c_{V}\left(x_{1}\right) \\
c_{\gamma}\left(x_{1}\right)
\end{array}\right] \widehat{d} \\
{\left[\begin{array}{l}
\delta_{e} \\
\Phi_{c}
\end{array}\right]=-B^{-1}\left(x_{1}\right)\left[\begin{array}{l}
f_{V} \\
f_{\gamma}
\end{array}\right] } & {\left[\begin{array}{l}
v_{V}+k_{V} \operatorname{sat}\left(\frac{s_{V}(t)}{\Phi_{V}}\right) \\
v_{\gamma}+k_{\gamma} \operatorname{sat}\left(\frac{s_{\gamma}(t)}{\Phi_{\gamma}}\right)
\end{array}\right] . }
\end{aligned}
$$

Considering (10) and substituting (26) into (22), the augmented closed-loop system can be obtained as follows:

$$
\begin{gathered}
\dot{s}_{V}(t)=-k_{V} \operatorname{sat}\left(\frac{s_{V}(t)}{\Phi_{V}}\right)+c_{V}\left(x_{1}\right) e_{d}(t)+d_{V}, \\
\dot{s}_{\gamma}(t)=-k_{\gamma} \operatorname{sat}\left(\frac{s_{\gamma}(t)}{\Phi_{\gamma}}\right)+c_{\gamma}\left(x_{1}\right) e_{d}(t)+d_{\gamma}, \\
\dot{e}_{d}(t)=-l_{4}(x) e_{d}(t)+\dot{d} .
\end{gathered}
$$

Next, uniform ultimate boundedness (UUB) of the composite system (28) will be considered.

Theorem 6. For the composite system (28), if the sliding mode gains, $k_{V}$ and $k_{\gamma}$, satisfy

$$
\begin{gathered}
k_{V}>\operatorname{Max}\left\{0.25 \Phi_{V}\left(\beta_{V 1}^{-2} \sigma_{V 1}^{2}+\beta_{V 2}^{-2}\right),(1+\beta) \sigma \sigma_{V 1}+\sigma_{V 2}\right\}, \\
k_{\gamma}>\operatorname{Max}\left\{0.25 \Phi_{\gamma}\left(\beta_{\gamma 1}^{-2} \sigma_{\gamma 1}^{2}+\beta_{\gamma 2}^{-2}\right),(1+\beta) \sigma \sigma_{\gamma 1}+\sigma_{\gamma 2}\right\}
\end{gathered}
$$

and the fourth element of observer gain, $l_{4}(x)$, is chosen as $l_{0}(1+$ $M(x))$ with the constant $l_{0}$ satisfying

$$
l_{0}>\frac{\left(1+\beta_{V 1}^{2}+\beta_{\gamma 1}^{2}\right)}{[2(1+\underline{M})]},
$$

then the solutions of composite system (28) are UUB, where positive constants $\beta_{V 1}, \beta_{\gamma 1}, \beta, \beta_{V 2}$, and $\beta_{\gamma_{2}}$ are tuning parameters to obtain the appropriate values of control gains, and positive constants $\sigma_{V 1}, \sigma_{\gamma 1}, \sigma, \sigma_{V 2}$, and $\sigma_{\gamma 2}$ are the upper bounds of $c_{V}\left(x_{1}\right), c_{\gamma}\left(x_{1}\right), \dot{d}, d_{V}$, and $d_{\gamma}$; that is, $\left|c_{V}\left(x_{1}\right)\right| \leq \sigma_{V 3}$, $\left|c_{\gamma}\left(x_{1}\right)\right| \leq \sigma_{\gamma 3},|\dot{d}| \leq \sigma,\left|d_{V}\right| \leq \sigma_{V 2}$, and $\left|d_{\gamma}\right| \leq \sigma_{\gamma 2}$.
Proof. A Lyapunov function candidate for the system (28) is chosen as $W(t)=W_{d}(t)+W_{s}(t)$, where $W_{d}(t)=e_{d}^{2}(t)$ and $W_{s}(t)=(1 / 2)\left(s_{V}^{2}(t)+s_{\gamma}^{2}(t)\right)$. According to Theorem $3, \dot{W}_{d}(t) \leq$ $-\left[2 l_{0}(1+M(x))-1\right] e_{d}^{2}(t)+\sigma^{2}$.

Taking the derivative of $W_{s}(t)$ along the trajectories of (28), we have

$$
\begin{aligned}
\dot{W}_{s}(t)= & s_{V}(t) \dot{s}_{V}(t)+s_{\gamma}(t) \dot{s}_{\gamma}(t) \\
= & s_{V}(t)\left[-k_{V} \operatorname{sat}\left(\frac{s_{V}(t)}{\Phi_{V}}\right)+c_{V}\left(x_{1}\right) e_{d}(t)+d_{V}\right] \\
& +s_{\gamma}(t)\left[-k_{\gamma} \operatorname{sat}\left(\frac{s_{\gamma}(t)}{\Phi_{\gamma}}\right)+c_{\gamma}\left(x_{1}\right) e_{d}(t)+d_{\gamma}\right] .
\end{aligned}
$$

When $\left|s_{V}(t) / \Phi_{V}\right| \leq 1$ and $\left|s_{\gamma}(t) / \Phi_{\gamma}\right| \leq 1$, the derivative of $W_{s}(t)$ can be expressed as

$$
\begin{aligned}
\dot{W}_{s}(t)= & -\left(\frac{k_{V}}{\Phi_{V}}\right) s_{V}^{2}(t)+c_{V}\left(x_{1}\right) e_{d}(t) s_{V}(t)+d_{V} s_{V}(t) \\
& -\left(\frac{k_{\gamma}}{\Phi_{\gamma}}\right) s_{\gamma}^{2}(t)+c_{\gamma}\left(x_{1}\right) e_{d}(t) s_{\gamma}(t)+d_{\gamma} s_{\gamma}(t) \\
= & -\left[\frac{k_{V}}{\Phi_{V}}-0.25 \beta_{V 1}^{-2} c_{V}^{2}\left(x_{1}\right)-0.25 \beta_{V 2}^{-2}\right] s_{V}^{2}(t) \\
& -\left[\frac{k_{\gamma}}{\Phi_{\gamma}}-0.25 \beta_{\gamma 1}^{-2} c_{\gamma}^{2}\left(x_{1}\right)-0.25 \beta_{\gamma 2}^{-2}\right] s_{\gamma}^{2}(t) \\
& -\left(0.5 \beta_{V 1}^{-1} c_{V}\left(x_{1}\right) s_{V}(t)-\beta_{V 1} e_{d}(t)\right)^{2} \\
& -\left(0.5 \beta_{V 2}^{-1} s_{V}(t)-\beta_{V 2} d_{V}\right)^{2} \\
& -\left(0.5 \beta_{\gamma 1}^{-1} c_{\gamma}\left(x_{1}\right) s_{\gamma}(t)-\beta_{\gamma 1} e_{d}(t)\right)^{2} \\
& -\left(0.5 \beta_{\gamma 2}^{-1} s_{\gamma}(t)-\beta_{\gamma 2} d_{\gamma}\right)^{2} \\
& +\left(\beta_{V 1}^{2}+\beta_{\gamma 1}^{2}\right) e_{d}^{2}(t)+\beta_{V 2}^{2} d_{V}^{2}+\beta_{\gamma 2}^{2} d_{\gamma}^{2} .
\end{aligned}
$$

Furthermore, the derivative of $W(t)$ along the trajectories of (28) can be given by

$$
\begin{aligned}
\dot{W}(t) \leq & -\left[\frac{k_{V}}{\Phi_{V}}-0.25 \beta_{V 1}^{-2} \sigma_{V 1}^{2}-0.25 \beta_{V 2}^{-2}\right] s_{V}^{2}(t) \\
& -\left[\frac{k_{\gamma}}{\Phi_{\gamma}}-0.25 \beta_{\gamma 1}^{-2} \sigma_{\gamma 1}^{2}-0.25 \beta_{\gamma 2}^{-2}\right] s_{\gamma}^{2}(t) \\
& -\left[2 l_{0}(1+M(x))-\left(1+\beta_{V 1}^{2}+\beta_{\gamma 1}^{2}\right)\right] e_{d}^{2}(t) \\
& +\beta_{V 2}^{2} \sigma_{V 2}^{2}+\beta_{\gamma 2}^{2} \sigma_{\gamma 2}^{2}+\sigma^{2}
\end{aligned}
$$




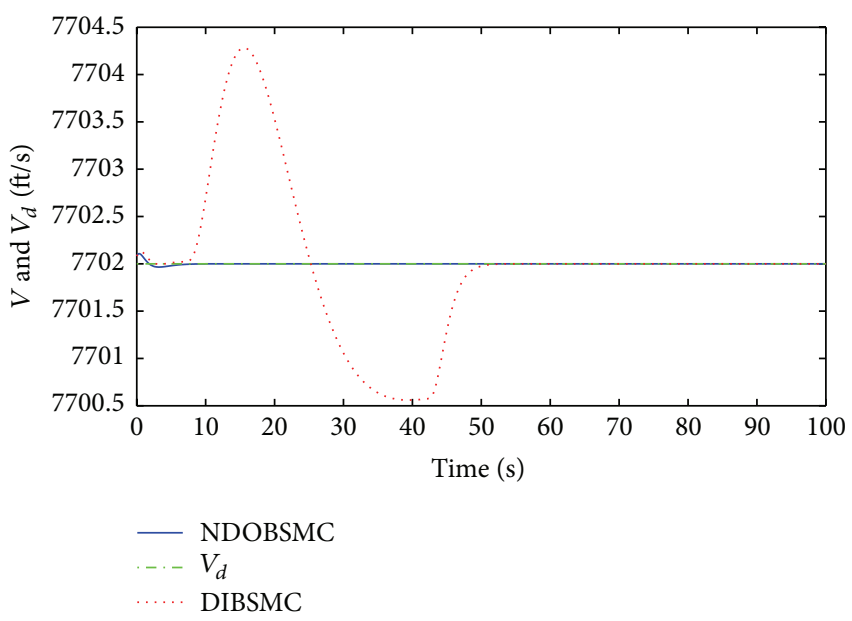

(a)

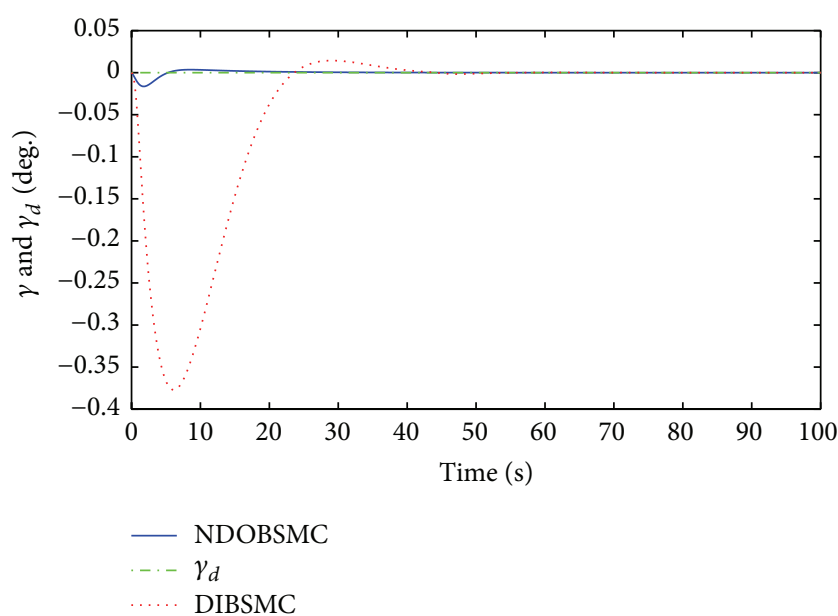

(c)

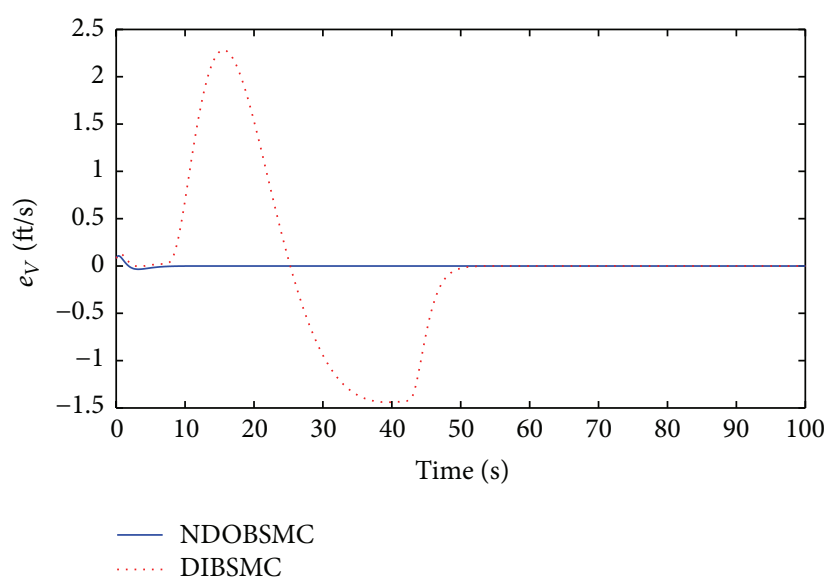

(b)

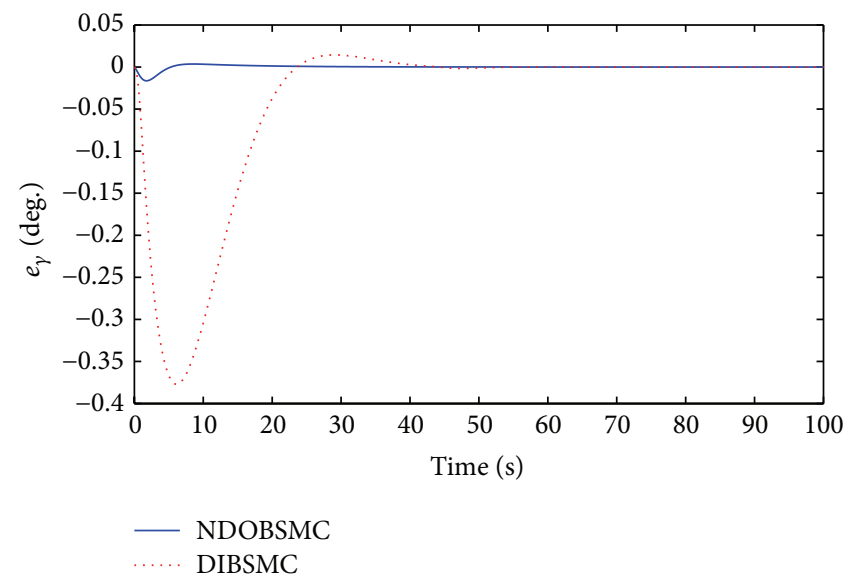

(d)

Figure 1: Case study 1: (a) velocity $V$ and desired signal $V_{d}$, (b) tracking error of velocity $e_{V}$, (c) flight path angle $\gamma$ and desired signal $\gamma_{d}$, and (d) tracking error of flight path angle $e_{\gamma}$.

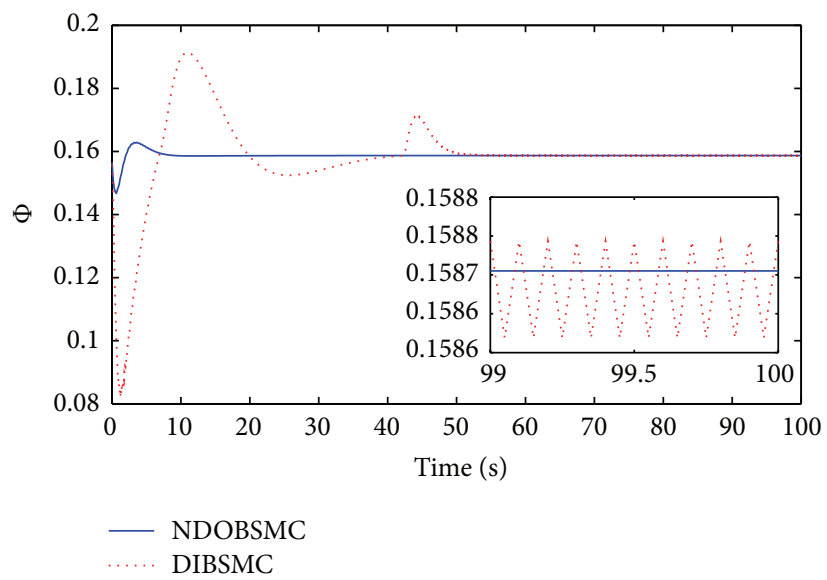

(a)

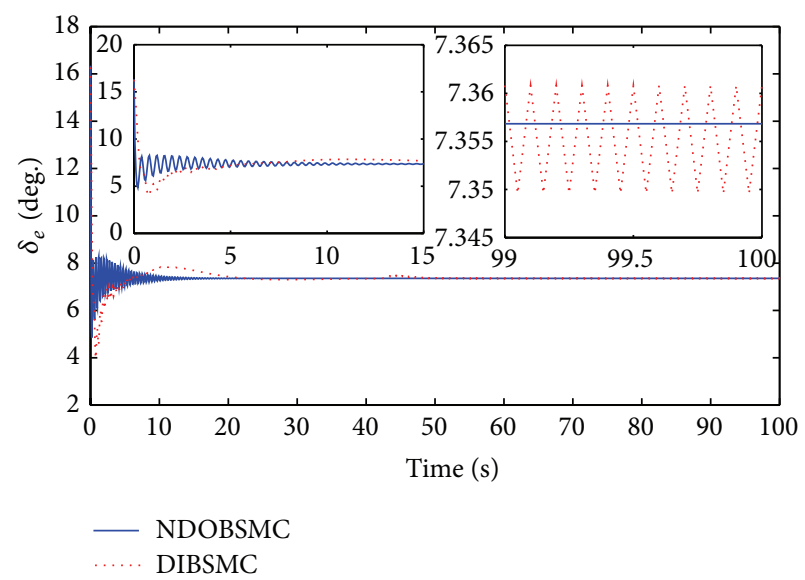

(b)

Figure 2: Case study 1: (a) fuel equivalence ratio $\Phi$, (b) elevator deflection $\delta_{e}$. 


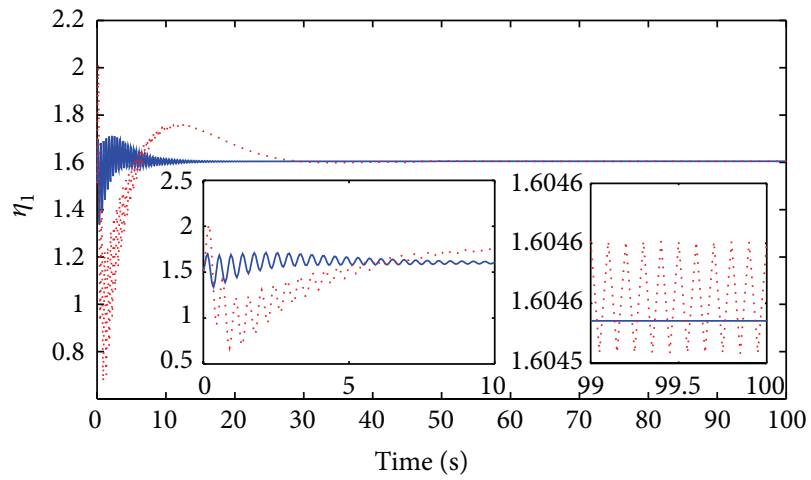

(a)

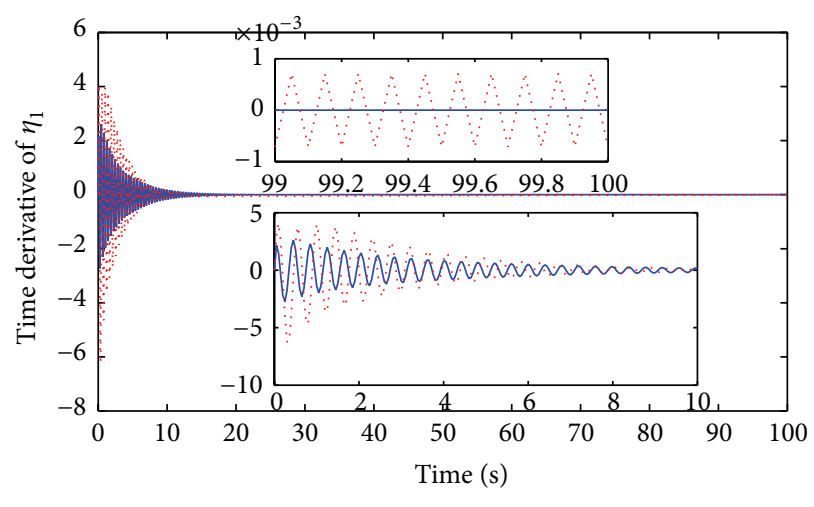

NDOBSMC

DIBSMC

(c)

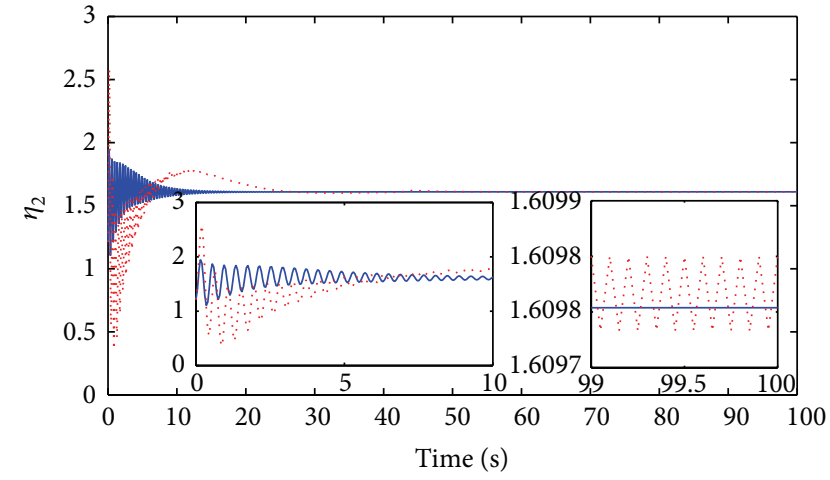

(b)

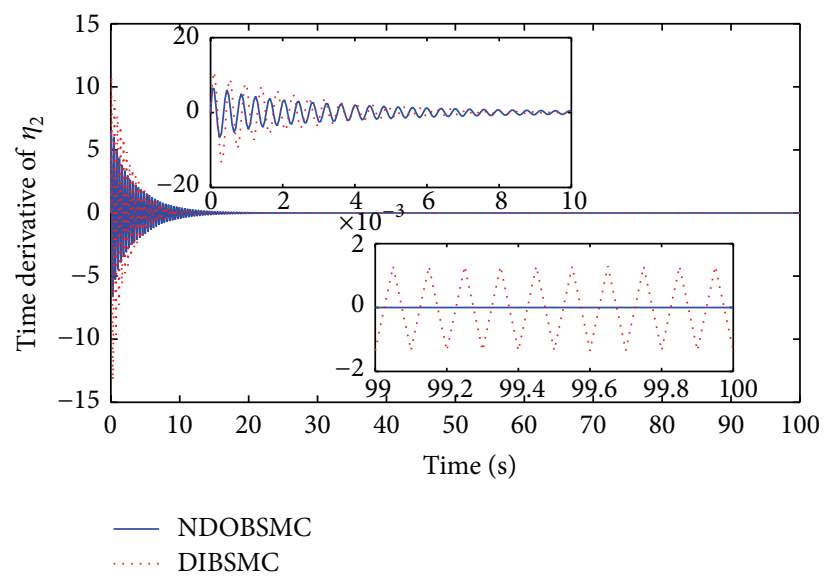

(d)

Figure 3: Case study 1: (a) flexible mode $\eta_{1}$, (b) flexible mode $\eta_{2}$, (c) $\dot{\eta}_{1}(t)$, and (d) $\dot{\eta}_{2}(t)$.

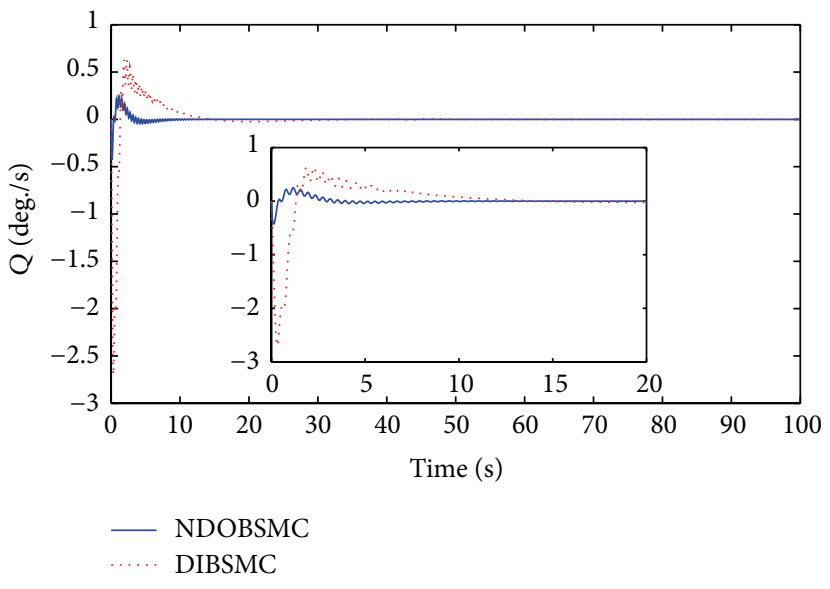

(a)

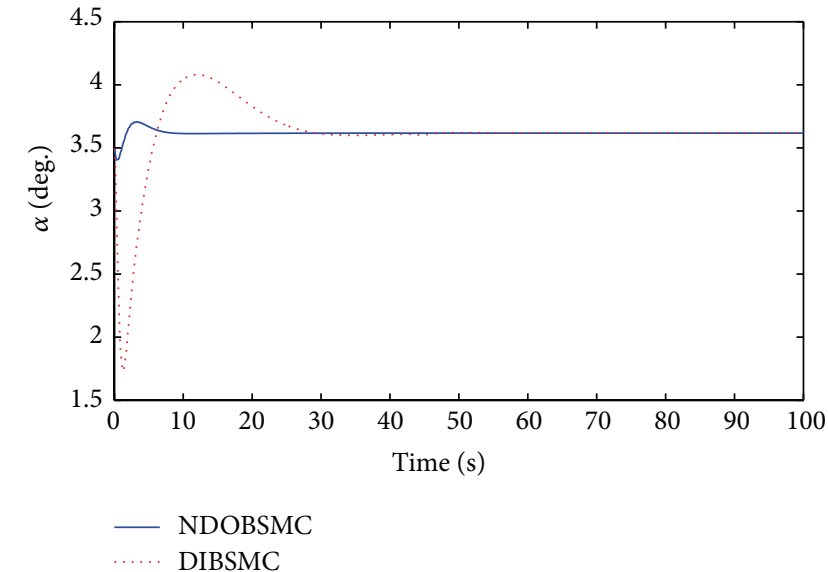

(b)

FIgURe 4: Case study 1: (a) pitch rate $Q$, (b) angle of attack $\alpha$. 
When $\left|s_{V}(t) / \Phi_{V}\right|>1$ and $\left|s_{\gamma}(t) / \Phi_{\gamma}\right|>1$, the derivative of $W_{s}(t)$ can be given by

$$
\begin{aligned}
\dot{W}_{s}(t)= & -k_{V}\left|s_{V}(t)\right|+c_{V}\left(x_{1}\right) e_{d}(t) s_{V}(t)+d_{V} s_{V}(t) \\
& -k_{\gamma}\left|s_{\gamma}(t)\right|+c_{\gamma}\left(x_{1}\right) e_{d}(t) s_{\gamma}(t)+d_{\gamma} s_{\gamma}(t) \\
\leq & -\left(k_{V}-\left|c_{V}\left(x_{1}\right)\right|\left|e_{d}(t)\right|-\left|d_{V}\right|\right)\left|s_{V}(t)\right| \\
& -\left(k_{\gamma}-\left|c_{\gamma}\left(x_{1}\right)\right|\left|e_{d}(t)\right|-\left|d_{\gamma}\right|\right)\left|s_{\gamma}(t)\right| .
\end{aligned}
$$

By now, the derivative of $W(t)$ along the trajectories of (28) becomes

$$
\begin{aligned}
\dot{W}(t) \leq & -\left(k_{V}-(1+\beta) \sigma \sigma_{V 1}-\sigma_{V 2}\right)\left|s_{V}(t)\right| \\
& -\left(k_{\gamma}-(1+\beta) \sigma \sigma_{\gamma 1}-\sigma_{\gamma 2}\right)\left|s_{\gamma}(t)\right| \\
& -\left[2 l_{0}(1+M(x))-1\right] e_{d}^{2}(t)+\sigma^{2} .
\end{aligned}
$$

Thus it can be concluded from Lemma 2 that the solutions of the augmented closed-loop system (28) are uniformly ultimately bounded, if conditions (29) and (30) are satisfied.

Remark 7. The result of Theorem $6\left(l_{0}>\left(1+\beta_{V}^{2}+\beta_{\gamma}^{2}\right) /[2(1+\right.$ $\underline{M})])$ is different from the result of Theorem $3\left(l_{0}>1 /[2(1+\right.$ $\underline{M})]$ ) because of the couplings between tracking errors and disturbance estimate error. So it is necessary to analyze the stability of the composite system (28).

\section{Simulation Studies}

The controller derived in the previous section will be tested in simulation using the full nonlinear model of FAHVs. Two case studies will be presented in this section. Firstly, the flexible effects on the pitching moment and the estimating performance of the disturbance observer are studied for the nominal model without parameter uncertainties and external disturbances. Secondly, the tracking performance and robustness of the NDOBSMC (26) and the DIBSMC (27) are compared for the FAHV model with composite disturbance.

In the first case study, the nonlinear function $p(x)$ is taken as $l_{0}\left(Q+c Q^{3}\right)$, where $c \geq 0$. Thus the NDO gains $l(x)=\left[\begin{array}{llllll}0 & 0 & 0 & l_{0}\left(1+3 c Q^{2}\right) & 0 & 0\end{array}\right]$. The sliding mode gains of NDOBSMC are chosen as $k_{V}=10$ and $k_{\gamma}=0.01$, while the bigger sliding mode gains of DIBSMC are chosen as $k_{V}=16$ and $k_{\gamma}=0.02$ in order to attenuate the pitching disturbance moment, $\bar{d}_{f}$, which is produced by couplings between flexible and rigid modes. Figure 1 shows the simulation results of $V$, $\gamma, e_{V}$, and $e_{\gamma}$ using NDOBSMC and DIBSMC. The simulation results confirm the fact that the disturbance $\bar{d}_{f}$ affects the tracking performance of velocity and FPA. And it can be observed that the tracking errors of velocity and FPA under DIBSMC are greater than the ones under NDOBSMC, even if the sliding mode gains of DIBSMC are bigger than the ones of NDOBSMC. It is caused that the NDOBC is able to

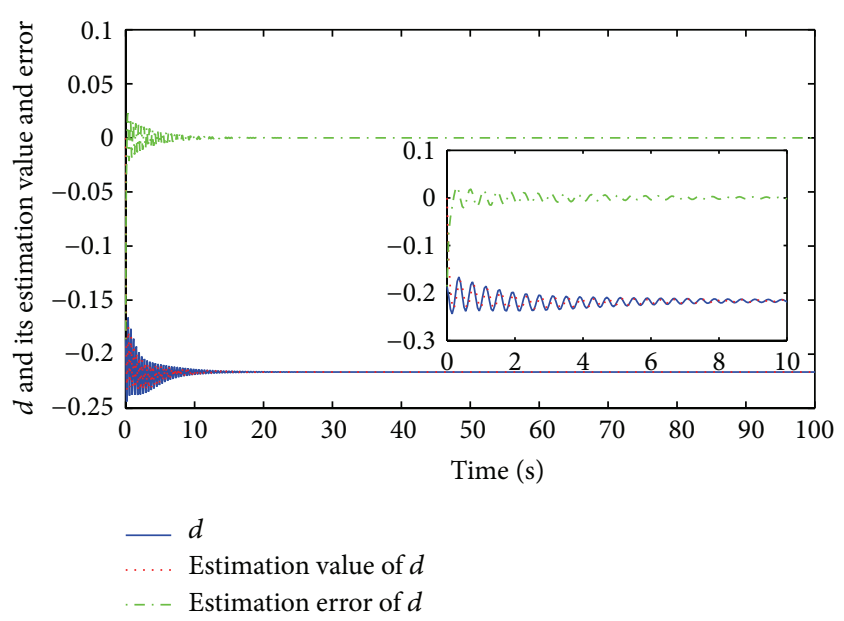

FIGURE 5: Case study 1: composite disturbance $d$, estimation value and error of $d$.

estimate and compensate for this disturbance $\bar{d}_{f}$, as shown in Figure 5. Furthermore, it can be seen from Figures 2, 3, 4 , and 5 that the changes of $\Phi_{c}, \delta_{e}, \eta_{1}, \eta_{2}, \dot{\eta}_{1}, \dot{\eta}_{2}, Q$, and $\alpha$ under DIBSMC are bigger than the ones under NDOBSMC. In addition, it is observed from Figures 2 and 3 that the chattering of $\Phi_{c}, \delta_{e}, \eta_{1}, \eta_{2}, \dot{\eta}_{1}$, and $\dot{\eta}_{2}$ using DIBSMC can be reduced using NDOBSMC. Finally, we can conclude that the effects produced by the disturbance $\bar{d}_{f}$ could be reduced using the NDOBSMC.

The second case study considers a maneuver task, that is, acceleration at constant dynamic pressure at time $t=$ $50 \mathrm{~s}$. Thus the concrete forms of command signals can be described as $V_{d}(t)=600 V_{d}^{0}(t)$ and $\gamma_{d}(t)=\arcsin \left(2 h_{s} \dot{V}_{d}(t) /\right.$ $\left.V_{d}^{2}(t)\right)$, where the condition $\bar{q}=$ const yields $\gamma_{d}(t)=$ $\arcsin \left(2 h_{s} \dot{V}_{d}(t) / V_{d}^{2}(t)\right)$ (details in [18]), $V_{d}^{0}(t)=1-(1 /$ $\left.\sqrt{1-\zeta^{2}}\right) e^{-\zeta \omega_{n} t} \sin \left(\omega_{d} t+\beta\right), \omega_{d}=\omega_{n} \sqrt{1-\zeta^{2}}, \beta=\arccos \zeta$, and $\zeta$ and $\omega_{n}$ are chosen as 0.95 and 0.025 , respectively. Furthermore, the pitching disturbance moment, $\bar{d}_{o}$, which originates from the following parameter uncertainties and external disturbances, is added in this case.

(1) When $t \in[0 \mathrm{~s}, 400 \mathrm{~s}]$, parameter uncertainties $\Delta C_{L}^{(\cdot)}=$ $\Delta C_{D}^{(\cdot)}=\Delta C_{M}^{(\cdot)}=\Delta C_{T}^{(\cdot)}=\Delta C_{T \Phi}^{(\cdot)}=\Delta C_{N_{i}}^{(\cdot)}=20 \%, i=$ 1,2 , are added in the simulation.

(2) When $t \in[0 \mathrm{~s}, 250 \mathrm{~s}]$, pitching disturbance moment, $4.6 \times 10^{4}(\sin (0.25 t))$, which is produced by external interferences, is added in the simulation.

Recall that $d=\left(\bar{d}_{f}+\bar{d}_{o}\right) / \bar{I}_{y y}$, where $\bar{d}_{o}$ is the pitching disturbance moment produced by the above parameter uncertainties and external interferences. Thus tracking performance and robustness of the NDOBSMC and the traditional DIBSMC are compared for the system with the composite disturbance $d$ in this case. In order to attenuate the composite disturbance, the sliding mode gains of DIBSMC are chosen as $k_{V}=20$ and $k_{\gamma}=0.18$, while the sliding mode gains of NDOBSMC are chosen as $k_{V}=14$ and $k_{\gamma}=0.05$. In addition, the nonlinear function $p(x)$ and NDO gain $l(x)$ are the same 


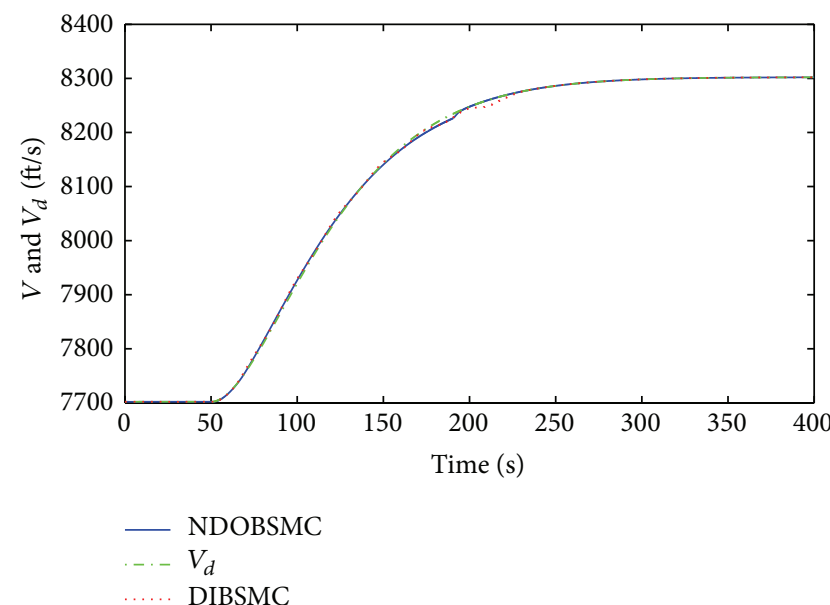

(a)

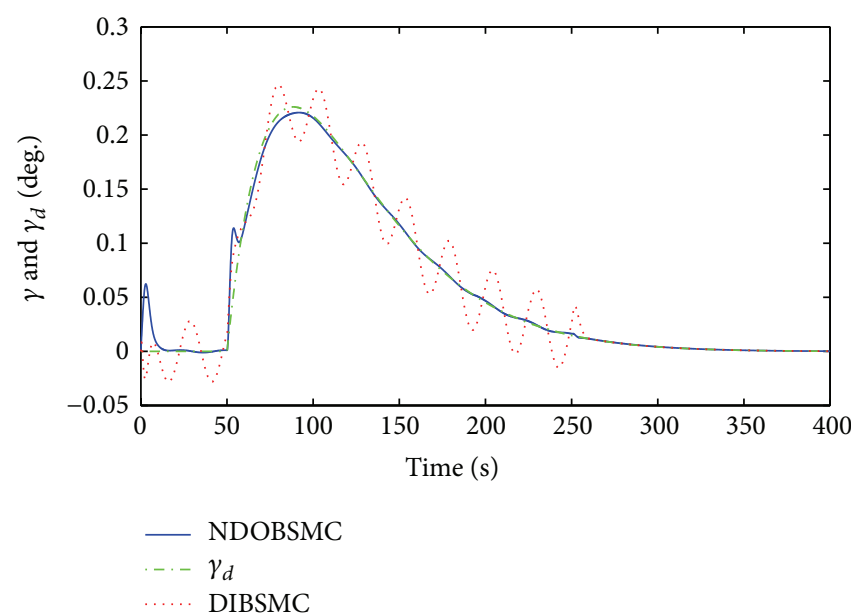

(c)

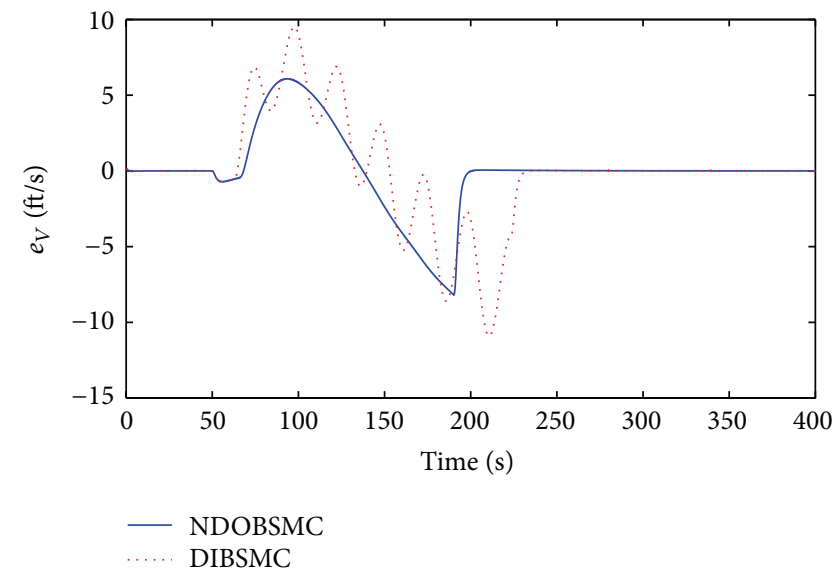

(b)

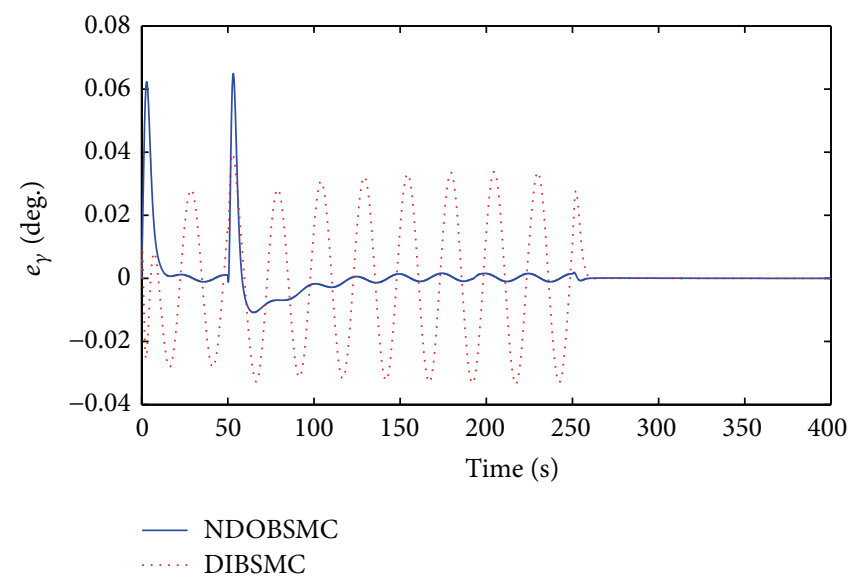

(d)

Figure 6: Case study 2: (a) velocity $V$ and desired signal $V_{d}$, (b) tracking error of velocity $e_{V}$, (c) flight path angle $\gamma$ and desired signal $\gamma_{d}$, and (d) tracking error of flight path angle $e_{\gamma}$.

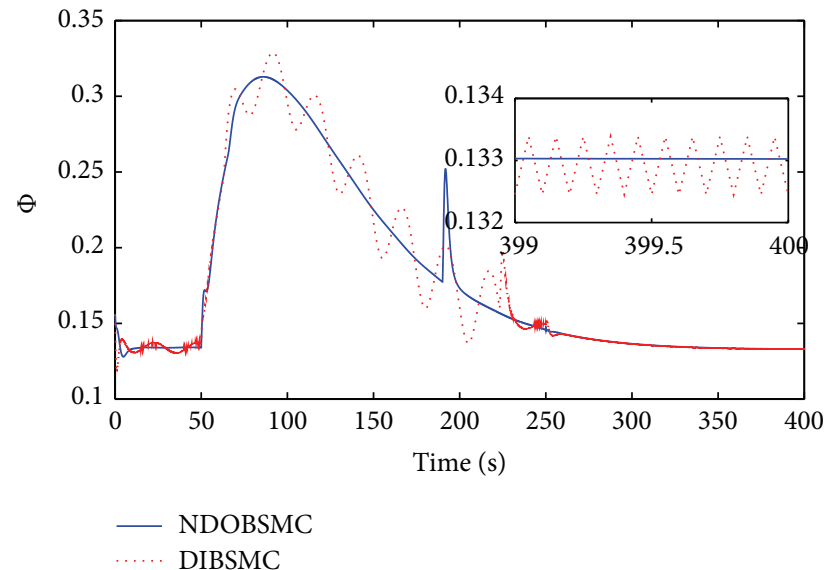

(a)

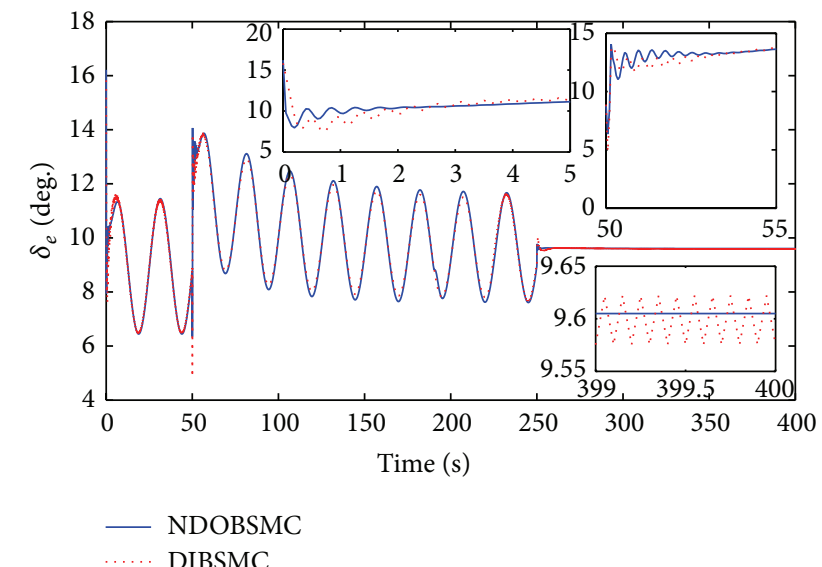

(b)

Figure 7: Case study 2: (a) fuel equivalence ratio $\Phi$, (b) elevator deflection $\delta_{e}$. 


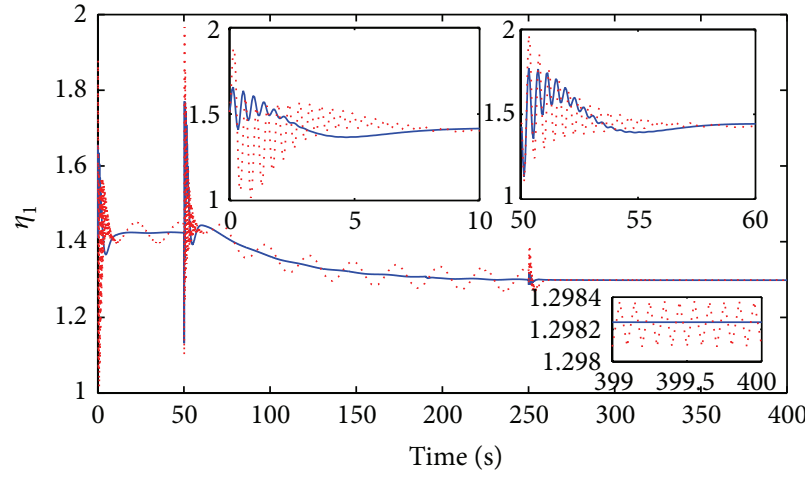

(a)

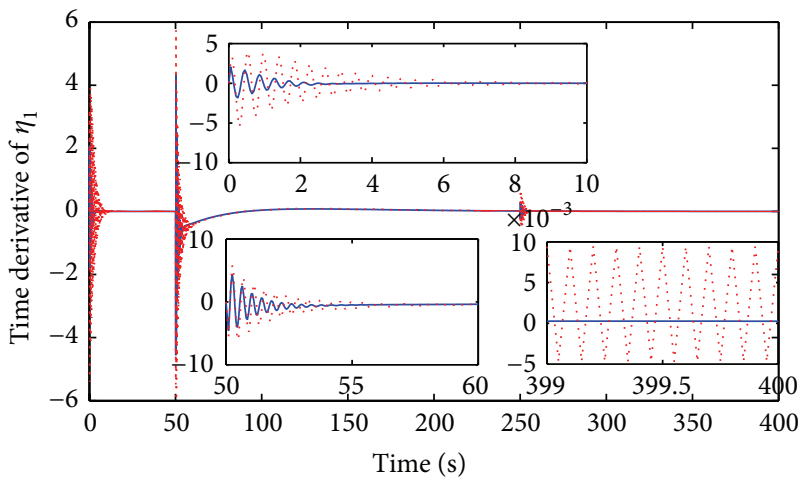

NDOBSMC

DIBSMC

(c)

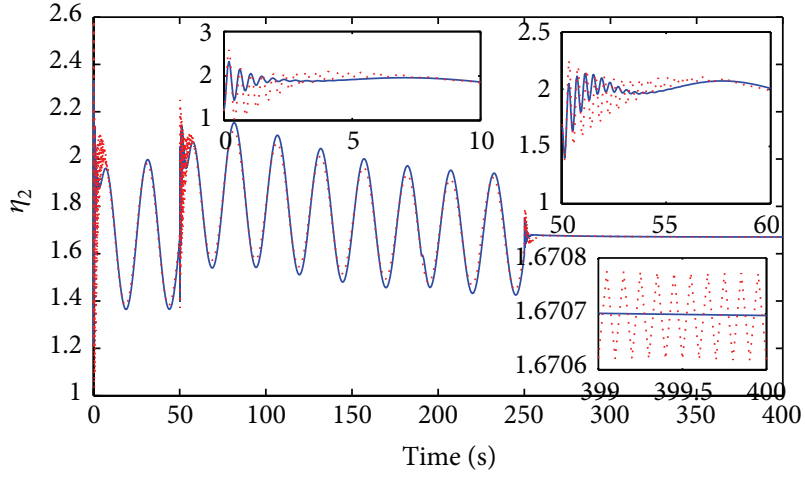

(b)

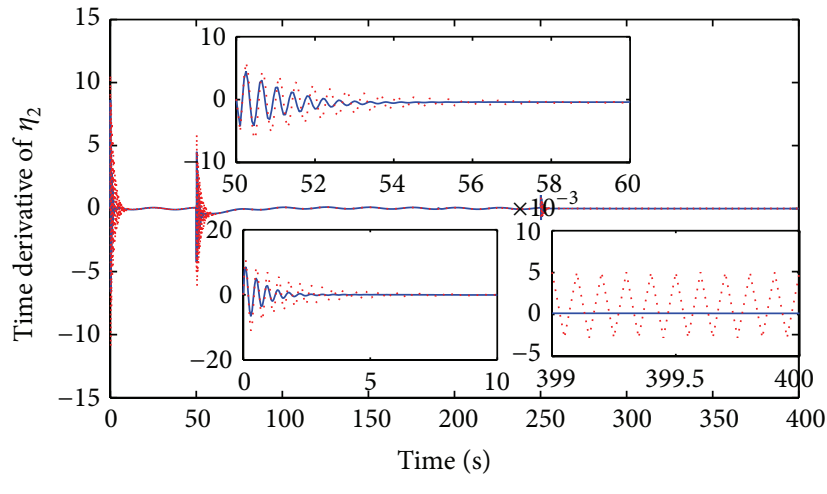

- NDOBSMC

DIBSMC

Figure 8: Case study 2: (a) flexible mode $\eta_{1}$, (b) flexible mode $\eta_{2}$, (c) $\dot{\eta}_{1}(t)$, and (d) $\dot{\eta}_{2}(t)$.

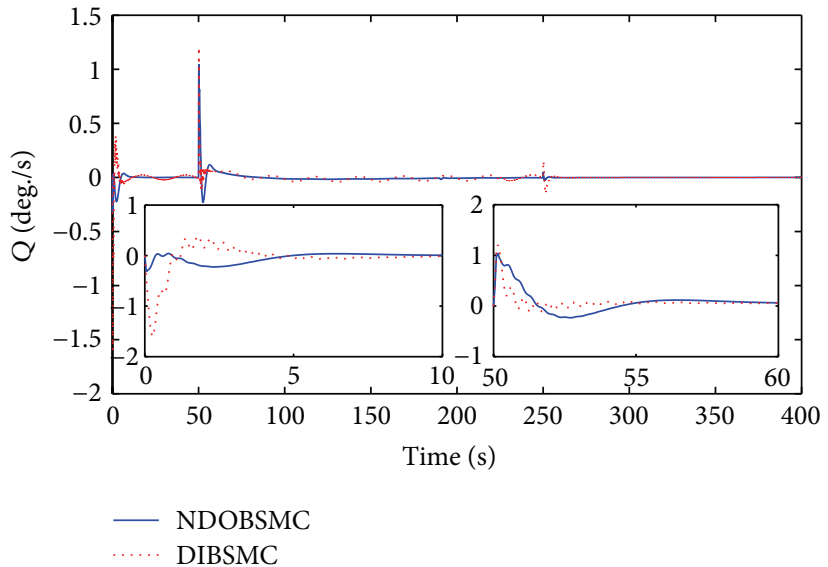

(a)

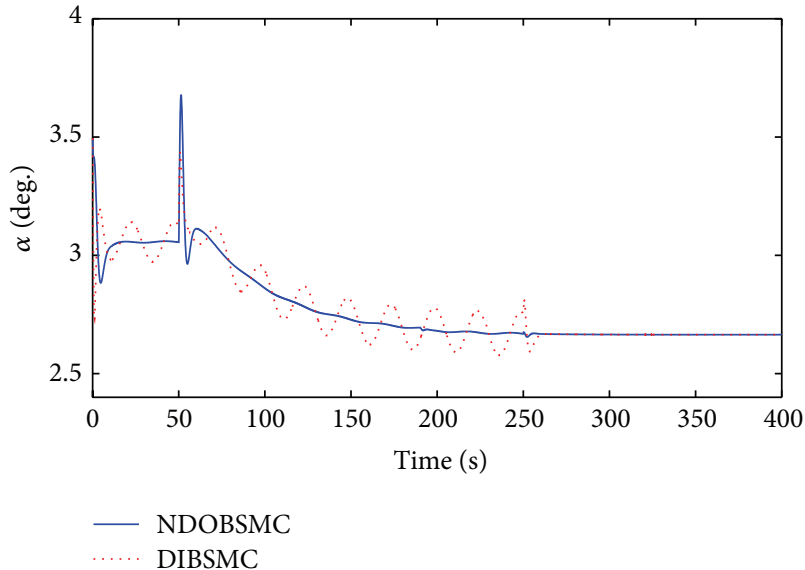

(b)

FIGURE 9: Case study 2: (a) pitch rate $Q$, (b) angle of attack $\alpha$.

as the first case. Figures 6 to 9 show the simulation results of $V, \gamma, e_{V}, e_{\gamma}, \Phi_{c}, \delta_{e}, \eta_{1}, \eta_{2}, \dot{\eta}_{1}, \dot{\eta}_{2}, Q$, and $\alpha$ using NDOBSMC and DIBSMC. It can be seen from Figure 6 that the tracking ability of velocity and FPA using NDOBSMC are better than the ones using traditional DIBSMC because the NDOBC can estimate and compensate for the composite disturbance $d$ shown in Figure 10. And it can be observed from Figures 7 and 8 that the chattering of traditional DIBSMC in presence of composite disturbances can be attenuated with the NDOBC. Furthermore, the changes of $\eta_{1} \eta_{2}, \dot{\eta}_{1}, \dot{\eta}_{2}, Q$, and 


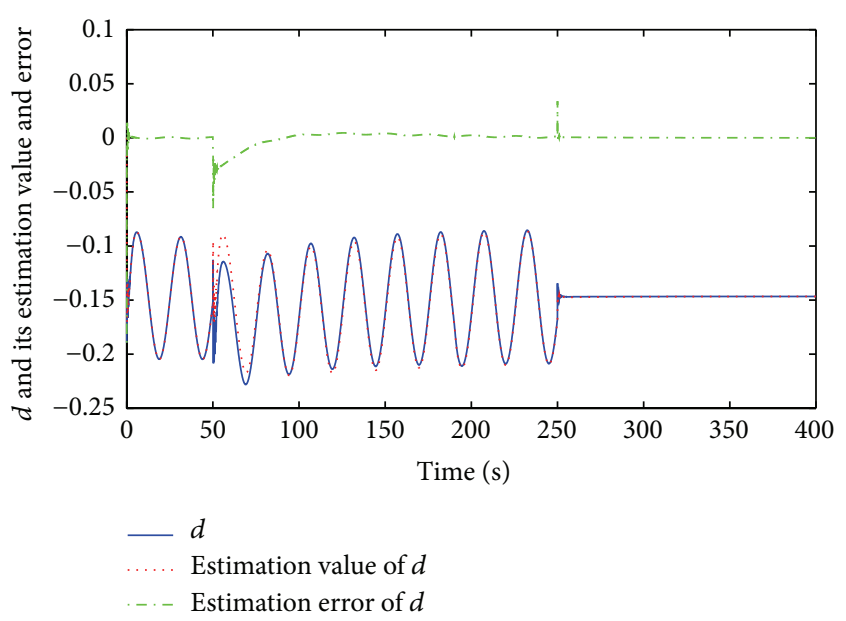

FIgURE 10: Case study 2: composite disturbance $d$, estimation value and error of $d$.

$\alpha$ are smaller using NDOBSMC, as shown in Figures 8 and 9. In a word, the tracking performance and robustness of NDOBSMC are better than those of DIBSMC.

\section{Conclusion}

In this paper, a nonlinear disturbance observer (NDO) based sliding mode controller (NDOBSMC) is designed for the nonlinear longitudinal model of flexible air-breathing hypersonic vehicles, and it is proved that the composite system is uniformly ultimately bounded when the controller gains and disturbance observer gains meet specified conditions. Simulation results show that the NDO based compensator can estimate and compensate for the composite disturbance produced by the couplings between flexible and rigid modes, parameter uncertainties, and external disturbances. Furthermore, tracking performance and robustness can be improved by using NDOBSMC, compared with the traditional dynamic inversion based sliding mode controller. One of the future research topics is to study the control problem for flexible air-breathing hypersonic vehicles based on the data-driven framework [26-28].

\section{Appendix}

The detailed expressions of $L^{0}, D^{0}, T^{0}, N_{i}^{0}, M^{0}, \Delta L, \Delta L, \Delta D$, $\Delta T, \Delta N_{i}$, and $\Delta M$ are given by

$$
\begin{aligned}
L^{0}= & \bar{q} S\left(C_{L^{0}}^{\alpha} \alpha+C_{L^{0}}^{0}+C_{L^{0}}^{\delta_{e}} \delta_{e}\right) \\
D^{0}= & \bar{q} S\left(C_{D^{0}}^{\alpha^{2}} \alpha^{2}+C_{D^{0}}^{\alpha} \alpha+C_{D^{0}}^{0}+C_{D^{0}}^{\delta_{e}^{2}} \delta_{e}^{2}+C_{D^{0}}^{\delta_{e}} \delta_{e}\right) \\
T^{0}= & \left(C_{T^{0} \Phi^{3}}^{\alpha^{3}}+C_{T^{0} \Phi^{\alpha^{2}}}^{2} \alpha^{2}+C_{T^{0} \Phi}^{\alpha} \alpha+C_{T^{0} \Phi}^{0}\right) \Phi \\
& \quad+C_{T^{0}}^{\alpha^{3}} \alpha^{3}+C_{T^{0}}^{\alpha^{2}} \alpha^{2}+C_{T^{0}}^{\alpha} \alpha+C_{T^{0}}^{0}, \\
& z^{0} T^{0}+\bar{q} S \bar{c}\left(C_{M^{0}}^{\alpha^{2}} \alpha^{2}+C_{M^{0}}^{\alpha} \alpha+C_{M^{0}}^{0}+C_{M^{0}}^{\delta_{e}} \delta_{e}\right)
\end{aligned}
$$

$$
\begin{aligned}
& N_{1}^{0}=C_{N_{1}^{0}}^{\alpha^{2}} \alpha^{2}+C_{N_{1}^{0}}^{\alpha} \alpha+C_{N_{1}^{0}}^{0}, \\
& N_{2}^{0}=C_{N_{2}^{0}}^{\alpha^{2}} \alpha^{2}+C_{N_{2}^{0}}^{\alpha} \alpha+C_{N_{2}^{0}}^{0}+C_{N_{2}^{0}}^{\delta_{e}} \delta_{e}, \\
& \Delta L=\bar{q} S\left(\Delta C_{L}^{\alpha} C_{L^{0}}^{\alpha} \alpha+\Delta C_{L}^{0} C_{L^{0}}^{0}+\Delta C_{L}^{\delta_{e}} C_{L^{0}}^{\delta_{e}} \delta_{e}\right), \\
& \Delta D=\bar{q} S\left(\Delta C_{D}^{\alpha^{2}} C_{D^{0}}^{\alpha^{2}} \alpha^{2}+\Delta C_{D}^{\alpha} C_{D^{0}}^{\alpha} \alpha+\Delta C_{D}^{0} C_{D^{0}}^{0}\right. \\
& \left.+\Delta C_{D}^{\delta_{e}^{2}} C_{D^{0}}^{\delta_{e}^{2}} \delta_{e}^{2}+\Delta C_{D}^{\delta_{e}} C_{D^{0}}^{\delta_{e}} \delta_{e}\right) \\
& \Delta T=\left(\Delta C_{T \Phi}^{\alpha^{3}} C_{T^{0} \Phi}^{\alpha^{3}} \alpha^{3}+\Delta C_{T \Phi}^{\alpha^{2}} C_{T^{0} \Phi}^{\alpha^{2}} \alpha^{2}\right. \\
& \left.+\Delta C_{T \Phi}^{\alpha} C_{T^{0} \Phi}^{\alpha} \alpha+\Delta C_{T \Phi}^{0} C_{T^{0} \Phi}^{0}\right) \Phi \\
& +\Delta C_{T}^{\alpha^{3}} C_{T^{0}}^{\alpha^{3}} \alpha^{3}+\Delta C_{T}^{\alpha^{2}} C_{T^{0}}^{\alpha^{2}} \alpha^{2} \\
& +\Delta C_{T}^{\alpha} C_{T^{0}}^{\alpha} \alpha+\Delta C_{T}^{0} C_{T^{0}}^{0} \text {, } \\
& \Delta M=z_{T} \Delta T+\bar{q} S \bar{c}\left(\Delta C_{M}^{\alpha^{2}} C_{M^{0}}^{\alpha^{2}} \alpha^{2}+\Delta C_{M}^{\alpha} C_{M^{0}}^{\alpha} \alpha\right. \\
& \left.+\Delta C_{M}^{0} C_{M^{0}}^{0}+\Delta C_{M}^{\delta_{e}} C_{M^{0}}^{\delta_{e}} \delta_{e}\right), \\
& \Delta N_{1}=\Delta C_{N_{1}}^{\alpha^{2}} C_{N_{1}^{0}}^{\alpha^{2}} \alpha^{2}+\Delta C_{N_{1}}^{\alpha} C_{N_{1}^{0}}^{\alpha} \alpha+\Delta C_{N_{1}}^{0} C_{N_{1}^{0}}^{0}, \\
& \Delta N_{2}=\Delta C_{N_{2}}^{\alpha^{2}} C_{N_{2}^{0}}^{\alpha^{2}} \alpha^{2}+\Delta C_{N_{2}}^{\alpha} C_{N_{2}^{0}}^{\alpha} \alpha+\Delta C_{N_{2}}^{0} C_{N_{2}^{0}}^{0} \\
& +\Delta C_{N_{2}}^{\delta_{e}} C_{N_{2}^{0}}^{\delta_{e}} \delta_{e} .
\end{aligned}
$$

The detailed expressions of $\sigma_{1}, \Sigma_{2}, \pi_{1}$, and $\Pi_{2}$ are given by

$$
\sigma_{1}=\left[\begin{array}{c}
-\frac{\partial \bar{D}^{0}}{\partial V} \\
\frac{\partial T^{0}}{\partial \alpha} \cos \alpha-T^{0} \sin \alpha-\frac{\partial \bar{D}^{0}}{\partial \alpha} \\
-m g \cos \gamma \\
\frac{\partial T^{0}}{\partial \Phi} \cos \alpha
\end{array}\right]^{T},
$$

$$
\pi_{1}=\left[\begin{array}{c}
-\frac{\partial^{2} \bar{L}^{0} / \partial V^{2}}{m V}-\frac{\bar{L}^{0}+T^{0} \sin \alpha}{m V^{2}}+\frac{g \cos \gamma}{V^{2}} \\
\frac{\partial \bar{L}^{0} / \partial \alpha+\left(\partial T^{0} / \partial \alpha\right) \sin \alpha+T^{0} \cos \alpha}{m V} \\
\frac{g \sin \gamma}{V} \\
\frac{\left(\partial T^{0} / \partial \alpha\right) \sin \alpha}{m V} \\
\Pi_{2}=\left[\begin{array}{lll}
\pi_{21} & \pi_{22} \pi_{23} \pi_{24}
\end{array}\right],
\end{array}\right.
$$

in which 


$$
\begin{aligned}
& \sigma_{21}=\left[\begin{array}{llll}
-\frac{\partial^{2} \bar{D}^{0}}{\partial V^{2}} & -\frac{\partial^{2} \bar{D}^{0}}{\partial \alpha \partial V} & 0 & 0
\end{array}\right], \\
& \sigma_{22}=\left[\begin{array}{c}
-\frac{\partial^{2} \bar{D}^{0}}{\partial \alpha \partial V} \\
\left(\frac{\partial^{2} T^{0}}{\partial \alpha^{2}}-T^{0}\right) \cos \alpha-2 \frac{\partial T^{0}}{\partial \alpha} \sin \alpha-\frac{\partial^{2} \bar{D}^{0}}{\partial \alpha^{2}} \\
0 \\
\frac{\partial^{2} T^{0}}{\partial \alpha \partial \Phi} \cos \alpha-\frac{\partial T^{0}}{\partial \Phi} \sin \alpha
\end{array}\right], \\
& \sigma_{23}=\left[\begin{array}{llll}
0 & 0 & m g \sin \gamma & 0
\end{array}\right]^{T}, \\
& \sigma_{24}=\left[\begin{array}{llll}
0 & \frac{\partial^{2} T^{0}}{\partial \Phi \partial \alpha} \cos \alpha-\frac{\partial T^{0}}{\partial \Phi} \sin \alpha & 0 & 0
\end{array}\right]^{T}, \\
& \pi_{21}=\left[\begin{array}{c}
-\frac{\partial^{2} \bar{L}^{0} / \partial V^{2}}{m V}-2 \frac{\partial \bar{L}^{0} / \partial V}{m V^{2}}+\frac{2\left(\bar{L}^{0}+T^{0} \sin \alpha\right)}{m V^{3}}-\frac{2 g \cos \gamma}{V^{3}} \\
\frac{\partial^{2} \bar{L}^{0} / \partial V \partial \alpha}{m V}-\frac{\partial \bar{L}^{0} / \partial \alpha+\left(\partial T^{0} / \partial \alpha\right) \sin \alpha+T^{0} \cos \alpha}{m V} \\
-\frac{g \sin \gamma}{V^{2}} \\
-\frac{\left(\partial T^{0} / \partial \alpha\right) \sin \alpha}{m V^{2}}
\end{array}\right], \\
& \pi_{22}=\left[\begin{array}{c}
-\frac{\partial^{2} \bar{L}^{0} / \partial V \partial \alpha}{m V}-\frac{\partial \bar{L}^{0} / \partial \alpha+\left(\partial T^{0} / \partial \alpha\right) \sin \alpha+T^{0} \cos \alpha}{m} \\
\frac{\partial^{2} \bar{L}^{0} / \partial \alpha^{2}+\left(\left(\partial^{2} T^{0} / \partial \alpha^{2}\right)-T^{0}\right) \sin \alpha+2\left(\partial T^{0} / \partial \alpha\right) \cos \alpha}{m V} \\
0 \\
-\frac{\left(\partial^{2} T^{0} / \partial \alpha \partial \Phi\right) \sin \alpha+\left(\partial T^{0} / \partial \Phi\right) \cos \alpha}{m V}
\end{array}\right],
\end{aligned}
$$

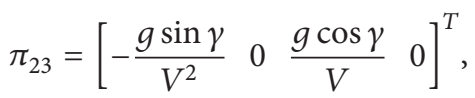

$$
\begin{aligned}
& \pi_{24}=\left[\begin{array}{c}
-\frac{\left(\partial T^{0} / \partial \Phi\right) \sin \alpha}{m V^{2}} \\
-\frac{\left(\partial^{2} T^{0} / \partial \alpha \partial \Phi\right) \sin \alpha+\left(\partial T^{0} / \partial \Phi\right) \cos \alpha}{m V} \\
0 \\
0
\end{array}\right] .
\end{aligned}
$$




\section{Nomenclature}

$h$ : Altitude

$V$ : Velocity

$\alpha$ : Angle of attack

$\gamma$ : Flight path angle

$\theta:$ Pitch angle, $\theta=\alpha-\gamma$

Q: Pitch rate, $\dot{\theta}=Q$

$\eta_{i}$ : Generalized elastic coordinate

$\widetilde{\psi}_{i}$ : Inertial coupling parameter

$\zeta_{i}$ : Damping ratio for $\eta_{i}$

$\omega_{i}$ : Natural frequency for $\eta_{i}$

$\delta_{e}$ : Elevator deflection

$\Phi: \quad$ Fuel equivalence ratio

$\zeta: \quad$ Damping ratio for $\Phi$

$\omega$ : Natural frequency for $\Phi$

L: $\quad$ Lift

D: Drag

T: Thrust

$N_{i}$ : Generalized elastic force

$M$ : Pitching moment

$I_{y y}$ : Moment of inertia

$m$ : Vehicle mass

$g$ : Acceleration due to gravity

$\rho:$ Density of air

$\bar{q}: \quad$ Dynamic pressure, $\bar{q}=\left(\rho V^{2}\right) / 2$

S: Reference area

$\bar{c}$ : Mean aerodynamic chord

$z_{T}$ : Thrust moment arm.

\section{Conflict of Interests}

The authors declare that there is no conflict of interests regarding the publication of this paper.

\section{Acknowledgments}

This work is supported by National Science Foundation of China Grants 61127007, 60925012, 61203041, and 91016004 and National 973 Program: 2012CB720003.

\section{References}

[1] E. T. Curran, "Scramjet engines: the first forty years," Journal of Propulsion and Power, vol. 17, no. 6, pp. 1138-1148, 2001.

[2] W. C. Engelund, "Hyper-X aerodynamics: the X-43A airframeintegrated scramjet propulsion flight-test experiments," Journal of Spacecraft and Rockets, vol. 38, no. 6, pp. 801-802, 2001.

[3] B. Fidan, M. Mirmirani, and P. A. Ioannou, "Flight dynamics and control of air-breathing hypersonic vehicles: review and new directions," in Proceedings of the 12th AIAA International Space Planes and Hypersonic Systems and Technologies, AIAA paper, no. 2003-7081, Norfolk, Va, USA, December 2003.

[4] M. A. Bolender, M. W. Oppenheimer, and D. B. Doman, "Effects of unsteady and viscous aerodynamics on the dynamics of a flexible air-breathing hypersonic vehicle," in Proceedings of the AIAA Atmospheric Flight Mechanics Conference, AIAA paper, no. 2007-6397, pp. 421-438, Hilton Head, SC, USA, August 2007.
[5] J. D. Shaughnessy, S. Z. Pinckney, J. D. McMinn, C. I. Cruz, and M. L. Kelley, "Hypersonic vehicle simulation model: wingedcone configuration," NASA TM 102610, 1990.

[6] M. A. Bolender and D. B. Doman, "Nonlinear longitudinal dynamical model of an air-breathing hypersonic vehicle," Journal of Spacecraft and Rockets, vol. 44, no. 2, pp. 374-387, 2007.

[7] D. O. Sigthorsson, P. Jankovsky, A. Serrani, S. Yurkovich, M. A. Bolender, and D. B. Doman, "Robust linear output feedback control of an airbreathing hypersonic vehicle," Journal of Guidance, Control, and Dynamics, vol. 31, no. 4, pp. 1052-1066, 2008.

[8] C. I. Marrison and R. F. Stengel, "Design of robust control systems for a hypersonic aircraft," Journal of Guidance, Control, and Dynamics, vol. 21, no. 1, pp. 58-63, 1998.

[9] Q. Wang and R. F. Stengel, "Robust nonlinear control of a hypersonic aircraft," Journal of Guidance, Control, and Dynamics, vol. 23, no. 4, pp. 577-585, 2000.

[10] H. Xu, M. D. Mirmirani, and P. A. Ioannou, "Adaptive sliding mode control design for a hypersonic flight vehicle," Journal of Guidance, Control, and Dynamics, vol. 27, no. 5, pp. 829-838, 2004.

[11] W. A. Butt, L. Yan, and A. S. Kendrick, "Robust adaptive dynamic surface control of a hypersonic flight vehicle," in Proceedings of the 49th IEEE Conference on Decision and Control (CDC '10), pp. 3632-3637, Atlanta, Ga, USA, December 2010.

[12] B. Xu, F. Sun, H. Liu, and J. Ren, "Adaptive Kriging controller design for hypersonic flight vehicle via back-stepping," IET Control Theory \& Applications, vol. 6, no. 4, pp. 487-497, 2012.

[13] Z. Gao, B. Jiang, P. Shi, J. Liu, and Y. Xu, "Passive fault-tolerant control design for near-space hypersonic vehicle dynamical system," Circuits, Systems, and Signal Processing, vol. 31, no. 2, pp. 565-581, 2012.

[14] K. P. Groves, D. O. Sigthorsson, A. Serrani, S. Yurkovich, M. A. Bolender, and D. B. Doman, "Reference command tracking for a linearized model of an air-breathing hypersonic vehicle," in Proceedings of the AIAA Guidance, Navigation, and Control Conference and Exhibit, pp. 2901-2914, San Francisco, Calif, USA, August 2005.

[15] X. Hu, L. Wu, C. Hu, and H. Gao, "Adaptive sliding mode tracking control for a flexible air-breathing hypersonic vehicle," Journal of the Franklin Institute, vol. 349, no. 2, pp. 559-577, 2012.

[16] X. Hu, L. Wu, C. Hu, and H. Gao, "Fuzzy guaranteed cost tracking control for a flexible air-breathing hypersonic vehicle," IET Control Theory \& Applications, vol. 6, no. 9, pp. 1238-1249, 2012.

[17] J. T. Parker, A. Serrani, S. Yurkovich, M. A. Bolender, and D. B. Doman, "Control-oriented modeling of an air-breathing hypersonic vehicle," Journal of Guidance, Control, and Dynamics, vol. 30, no. 3, pp. 856-869, 2007.

[18] L. Fiorentini, A. Serrani, M. A. Bolender, and D. B. Doman, "Nonlinear robust adaptive control of flexible air-breathing hypersonic vehicles," Journal of Guidance, Control, and Dynamics, vol. 32, no. 2, pp. 401-416, 2009.

[19] L. Guo and S. Y. Cao, "Anti-disturbance control theory for systems with multiple disturbances: a survey," ISA Transactions, vol. 53, no. 4, pp. 846-849, 2014.

[20] W.-H. Chen, "Nonlinear disturbance observer-enhanced dynamic inversion control of missiles," Journal of Guidance, Control, and Dynamics, vol. 26, no. 1, pp. 161-166, 2003.

[21] H. Liu, L. Guo, and Y. Zhang, "An anti-disturbance PD control scheme for attitude control and stabilization of flexible spacecrafts," Nonlinear Dynamics, vol. 67, no. 3, pp. 2081-2088, 2012. 
[22] M. Chen, C.-S. Jiang, and Q.-X. Wu, "Disturbance-observerbased robust flight control for hypersonic vehicles using neural networks," Advanced Science Letters, vol. 4, no. 4-5, pp. 1771$1775,2011$.

[23] S. H. Li, H. B. Sun, and C. Y. Sun, "Composite controller design for an air-breathing hypersonic vehicle," Proceedings of the IMechE, Part I: Journal of Systems and Control Engineering, vol. 69, no. 7, pp. 595-611, 2012.

[24] H. K. Khalil, Nonlinear Systems, Prentice-Hall, Upper Saddle River, NJ, USA, 3rd edition, 2002.

[25] J.-J. E. Slotine and W. Li, Applied Nonlinear Control, Prentice Hall, Upper Saddle River, NJ, USA, 1991.

[26] S. Yin, H. Luo, and S. X. Ding, "Real-time implementation of fault-tolerant control systems with performance optimization," IEEE Transactions on Industrial Electronics, vol. 61, no. 5, pp. 2402-2411, 2014.

[27] S. Yin, S. X. Ding, A. Haghani, H. Hao, and P. Zhang, "A comparison study of basic data-driven fault diagnosis and process monitoring methods on the benchmark Tennessee Eastman process," Journal of Process Control, vol. 22, no. 9, pp. 1567-1581, 2012.

[28] S. Yin, S. X. Ding, A. H. Sari, and H. Hao, "Data-driven monitoring for stochastic systems and its application on batch process," International Journal of Systems Science, vol. 44, no. 7, pp. 13661376, 2013. 


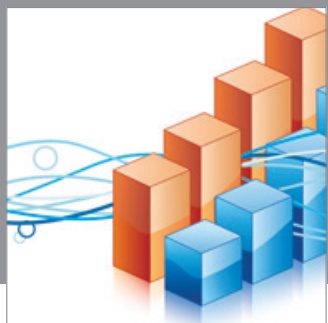

Advances in

Operations Research

mansans

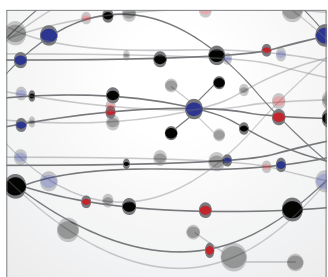

The Scientific World Journal
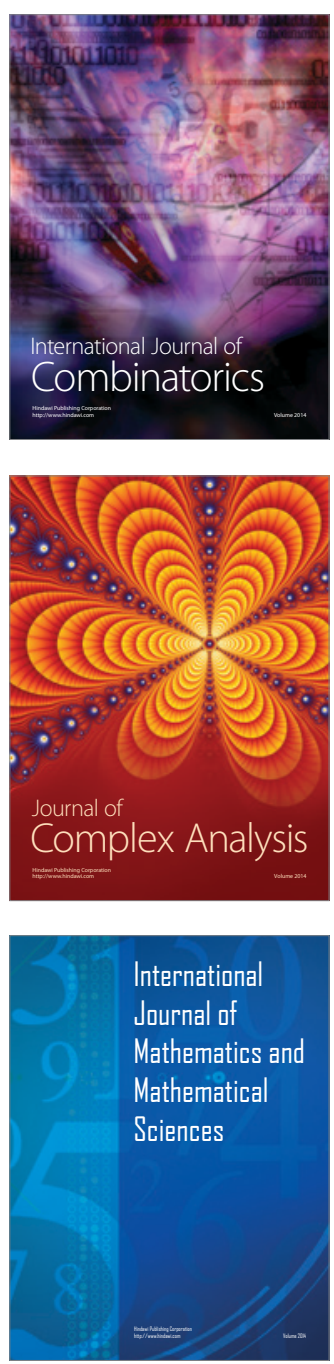
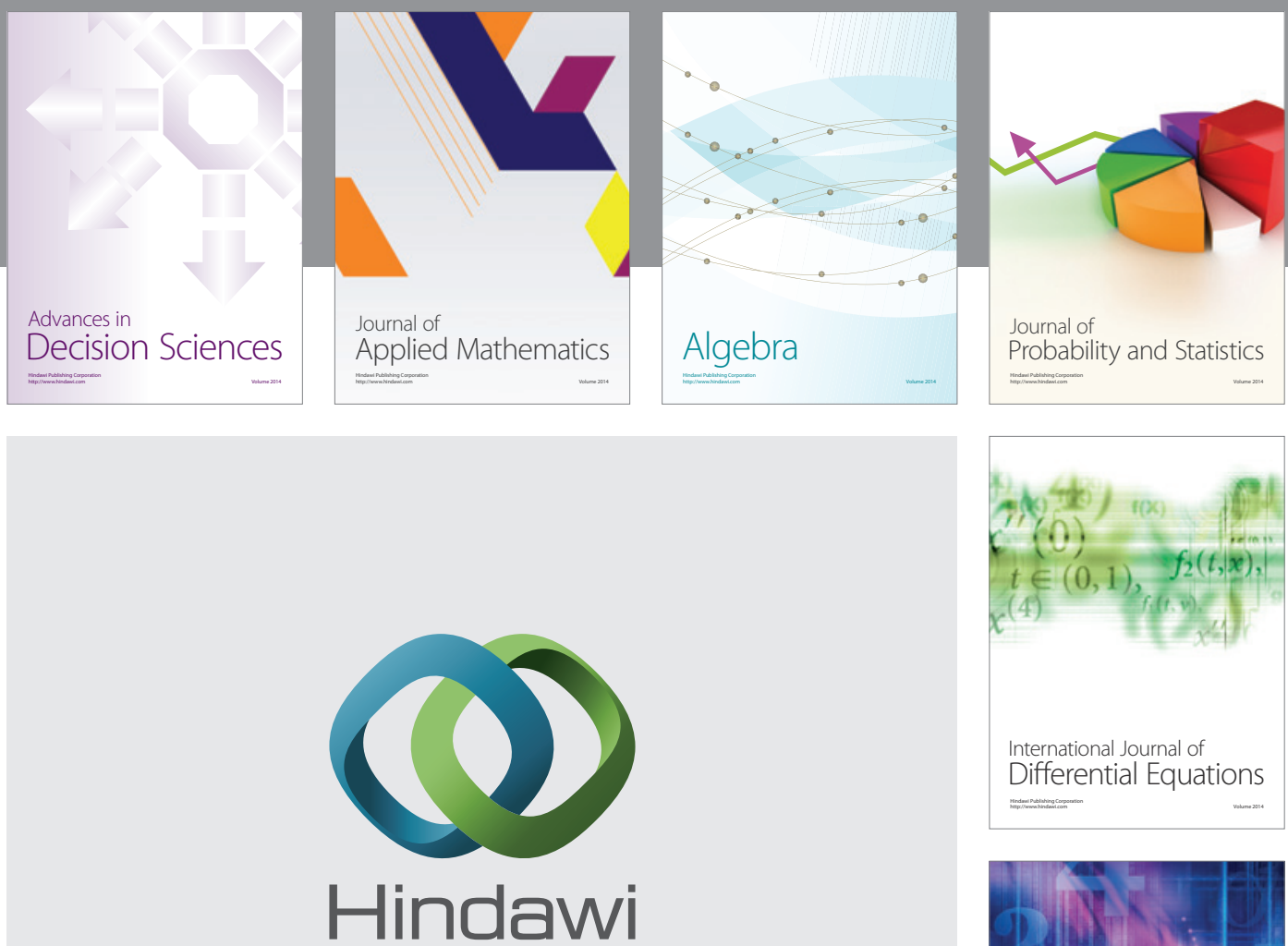

Submit your manuscripts at http://www.hindawi.com
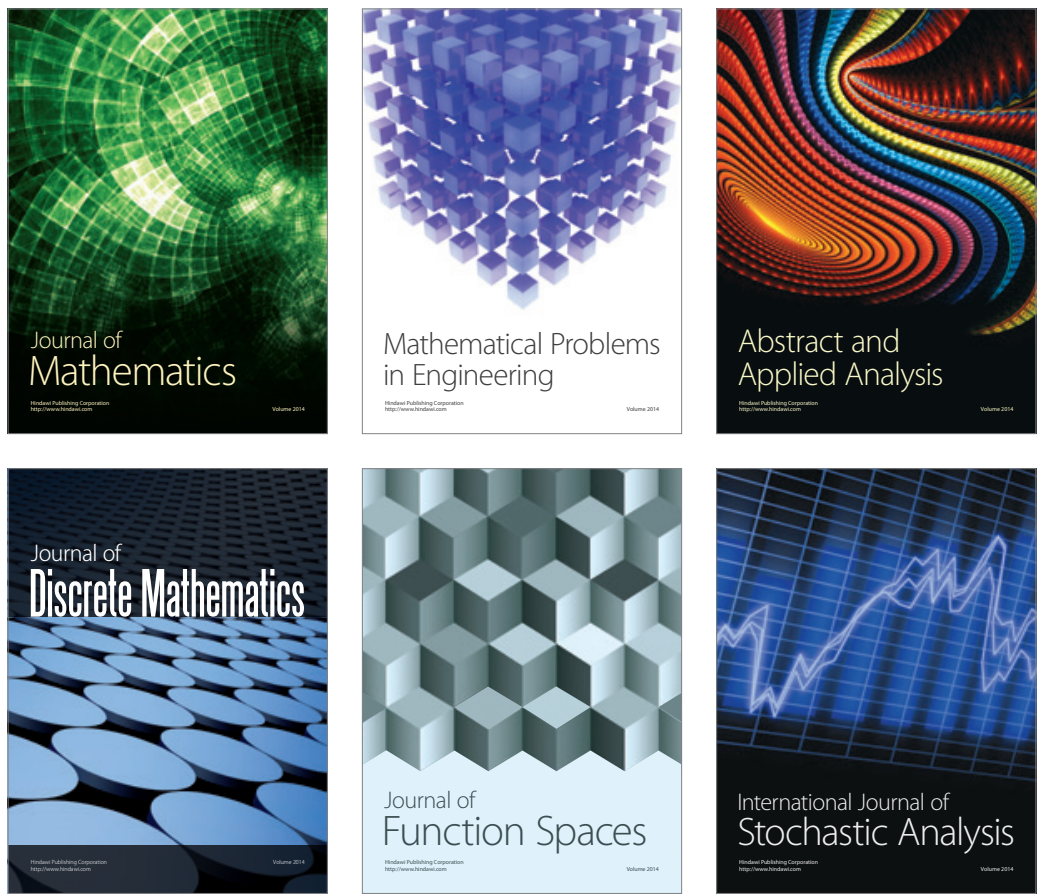

Journal of

Function Spaces

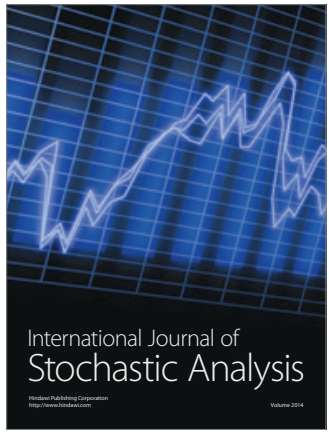

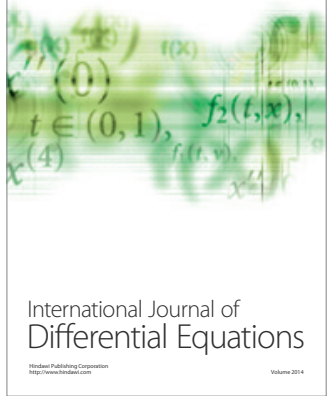
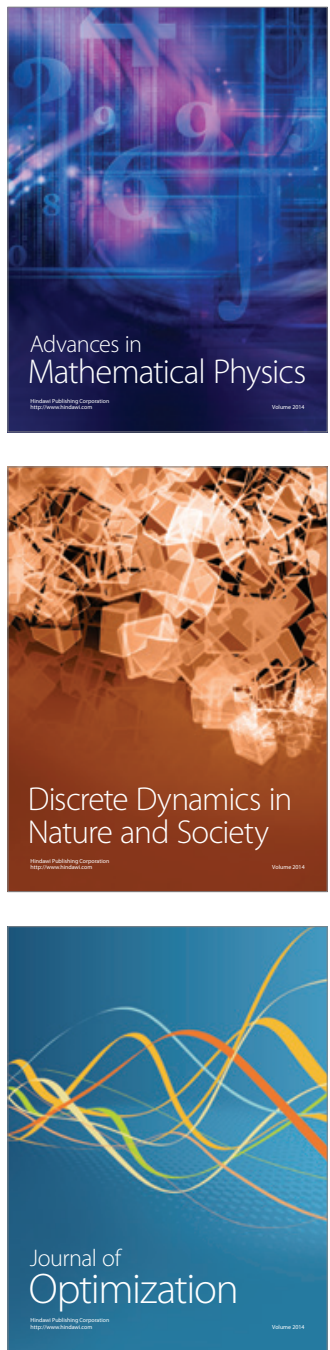